\title{
Urban-Rural disparities in status of hypertension in northeast China: a population-based study, 2017-2019
}

This article was published in the following Dove Press journal: Clinical Epidemiology

\author{
Liying Xing ${ }^{1,2}$ \\ Li Jing ${ }^{2}$ \\ Yuanmeng $\operatorname{Tian}^{2}$ \\ Min $\operatorname{Lin}^{3}$ \\ Zhi Du' \\ Han Yan ${ }^{2}$ \\ Guocheng Ren ${ }^{4}$ \\ Yingna Dong ${ }^{4}$ \\ Qun Sun ${ }^{5}$ \\ Shuang Liu ${ }^{6}$
}

'Department of Cardiology, The First Hospital of China Medical University, Shenyang II000I, People's Republic of China; ${ }^{2}$ Department of Chronic Disease, Liaoning Provincial Center for Disease Control and Prevention, Shenyang I 10005, People's Republic of China; ${ }^{3}$ Department of Cardiology, Central Hospital of Ben Xi City, Ben Xi, Liaoning, People's Republic of China; ${ }^{4}$ Department of Disease Control and Preventive, Central Hospital of Chao Yang City, Chaoyang, Liaoning, People's Republic of China; ${ }^{5}$ Department of Chronic Disease, Disease Control and Prevention of Chao Yang City, Chaoyang, Liaoning, People's Republic of China; ${ }^{6}$ Department of Cardiovascular Ultrasound, The First Hospital of China Medical University, Shenyang, Liaoning, People's Republic of China

Correspondence: Shuang Liu Department of Cardiovascular Ultrasound, First Hospital of China Medical University, No. 155 Nanjing

North Street, Shenyang II000I, Liaoning, People's Republic of China

Tel +86 I 3940340992

Fax +862423388556

Email liushuang_cmulh@163.com
Background: To investigate the status of hypertension and related risk factor disparities between urban and rural areas of northeast China.

Methods: A multi-stage, stratified, and cluster random sampling method was used to conduct the cross-sectional survey in Liaoning Province in 2017-2019. Finally, included 18,796 participants ( $28.9 \%$ urban, $71.1 \%$ rural) aged $\geq 40$ years. The prevalence and control rate of hypertension were estimated based on Chinese hypertension guidelines and the 2017 American College of Cardiology/American Heart Association (ACC/AHA) guidelines.

Results: The mean age of the population was $60.4 \pm 9.9$ years, and $61.0 \%$ were women. The overall prevalence of hypertension was $56.8 \%$. Compared to urban areas, hypertension was more prevalent, but the awareness, treatment, and control rates were lower in rural areas (59.2 vs $50.2,52.5 \%$ vs $47.0 \%, 46.9 \%$ vs $34.9 \%$, and $11.4 \%$ vs $3.7 \%, P<0.001$, respectively). Multivariate regression analysis identified that the lack of exercise (odds ratio (OR), 1.24; 95\% confidence interval (CI), 1.10-1.38) in rural areas, whereas overweight/obesity (OR, $2.01 ; 95 \%$ CI, 1.79-2.27) and alcohol consumption (OR, 1.20; 95\% CI, 1.01-1.41) in urban areas were specific risk factors for hypertension, besides common risk factors. Under the 2017 ACC/AHA guidelines, the prevalence of hypertension was $80.6 \%$ (urban $76.6 \%$, rural $82.2 \%$ ), increased 1.4-fold compared with the current Chinese guidelines, with increased rates of $27.9 \%$ in urban areas and $25.7 \%$ in rural areas.

Conclusion: A highly diverse prevalence for hypertension was found between urban and rural areas in northeast China. Region-specific strategies targeting the prevention and management of hypertension should be highlighted.

Keywords: hypertension, prevalence, control, urban, rural

\section{Introduction}

Cardiovascular disease has emerged as a serious public health problem and the leading cause of death and disability in China. ${ }^{1,2}$ As a major modifiable risk factor for cardiovascular disease, hypertension is responsible for nearly half of all cardiovascular morbidities and mortalities, and even small changes in blood pressure have substantial impact on the consequences of cardiovascular disease. ${ }^{3}$ Therefore, effective strategies focusing on prevention and management of hypertension should be emphasized.

The status of hypertension is associated with geographic, demographic, and socioeconomic characteristics. ${ }^{4}$ It is obvious that China has been experiencing rapid social and economic progress, and the epidemiologic characteristics of hypertension 
likely have changed concurrently, especially in rural populations. ${ }^{5}$ Moreover, China possesses the fourth-largest land area worldwide, and the status of its population is highly diverse among different regions. ${ }^{6}$ Although serial national surveys showed that the burden of hypertension increased dramatically over the past decade in China, the situation in northeast China seems more serious. ${ }^{7,8}$ Previous assessments of hypertension in northeast China are limited due to small or unrepresentative sample size, or insufficient estimation of related risk factors, particularly in urban areas. ${ }^{9,10}$ Furthermore, previous studies reported the steeper increase in prevalence of hypertension both in urban and rural areas in other countries; ${ }^{11,12}$ however, the current characteristics of hypertension as well as related risk factors in urban and rural areas of northeast China have not been well studied, and are still lacking.

Reliable epidemiologic data of the current burden of hypertension are crucial in formulating region-specific strategies, since previous studies suggested that China is lagging in risk factor management. ${ }^{13,14}$ In the present study, we conducted a cross-sectional survey of a large representative population in urban and rural areas of northeast China, aimed to profile the characteristics of hypertension and related risk factors for the future research directions on prevention and management strategies of hypertension in northeast China. Moreover, we performed a similar secondary analysis to determine the prevalence and control rate of hypertension according to the 2017 American College of Cardiology (ACC)/American Heart Association (AHA) High Blood Pressure Guideline, and estimated the burden of hypertension for both the urban and rural areas in northeast China.

\section{Methods}

\section{Study population}

The cross-sectional study was conducted in northeast China between September 2017 and March 2019. A multistage, stratified, and cluster random sampling method was used to ensure that the samples of the study were representative. Further details of the design are described in the following sections. In the first stage, four rural counties (Chaoyang, Lingyuan, Liaoyang, and Donggang) and three urban districts (Gongchangling, Liuerbao, Zhenan) were randomly selected from western, eastern, and central of Liaoning Province. Subsequently, 19 rural villages and 8 communities were randomly selected from these four counties and three cities. All permanent residents aged
40 years and above in each village $(n=22,009)$, except those who were pregnant or had a mental disorder, were eligible to participate; 18,796 (85.4\%) completed the study (Figure 1). The study was granted approval by the Central Ethics Committee at the China National Center for Cardiovascular Disease (Beijing, China). Written informed consent was obtained from all participants. The study was conducted in accordance with the Declaration of Helsinki.

\section{Data collection}

In brief, the demographic and clinical data were collected in a single clinic visit using a self-administered questionnaire. All eligible investigators were invited to attend the organized training and complete pilot interviews before the study was performed.

Fasting blood samples were collected after an overnight fast of $\geq 8 \mathrm{hrs}$ in the morning. Metabolic parameters including total cholesterol (TC), triglyceride (TG), low-density lipoprotein cholesterol (LDL-C), high-density lipoprotein cholesterol (HDL-C), fasting blood glucose (FBG), and glycosylated hemoglobin (HbAlc) were analyzed using an Abbott Diagnostics C800i auto-analyzer (Abbott Laboratories, Abbott Park, IL, USA) with commercial kits. Detailed methodology of the study is available elsewhere. ${ }^{15,16}$

\section{Definitions}

Hypertension was defined as a mean systolic blood pressure (SBP) $\geq 140 \mathrm{mmHg}$ or a mean diastolic blood pressure (DBP) $\geq 90 \mathrm{mmHg}$ and/or self-reported use of antihypertensive medication in the past 2 weeks according to the 2010 Chinese guidelines for the management of hypertension. ${ }^{17}$ Stage 1 of hypertension was defined as SBP 140-159 mmHg and/or DBP 90-99 $\mathrm{mmHg}$; stage 2 as $160-179 \mathrm{mmHg}$ and/or $100-109 \mathrm{mmHg}$; and stage 3 as $\geq 180 \mathrm{mmHg}$ and/or $\geq 110 \mathrm{mmHg}$. Prehypertension was defined as SBP $\geq 120 \mathrm{mmHg}$ and $<140 \mathrm{mmHg}$ and/or DBP $\geq 80 \mathrm{mmHg}$ and $<90 \mathrm{mmHg}$ and not being on antihypertensive medication. In addition, according to the 2017 ACC/AHA high blood pressure guidelines, the prevalence ( $\mathrm{SBP} \geq 130 \mathrm{mmHg}$ or $\mathrm{DBP} \geq 80 \mathrm{mmHg}$ ) and control (SBP $<130 \mathrm{mmHg}$ or $\mathrm{DBP}<80 \mathrm{mmHg}$ ) rate of hypertension were also estimated as a post hoc analysis. ${ }^{18}$

Awareness of hypertension was defined as self-report of any prior diagnosis hypertension by an eligible physician among the hypertensive population, treatment of hypertension was defined as use of antihypertensive medication in the past 2 weeks, and control of hypertension 


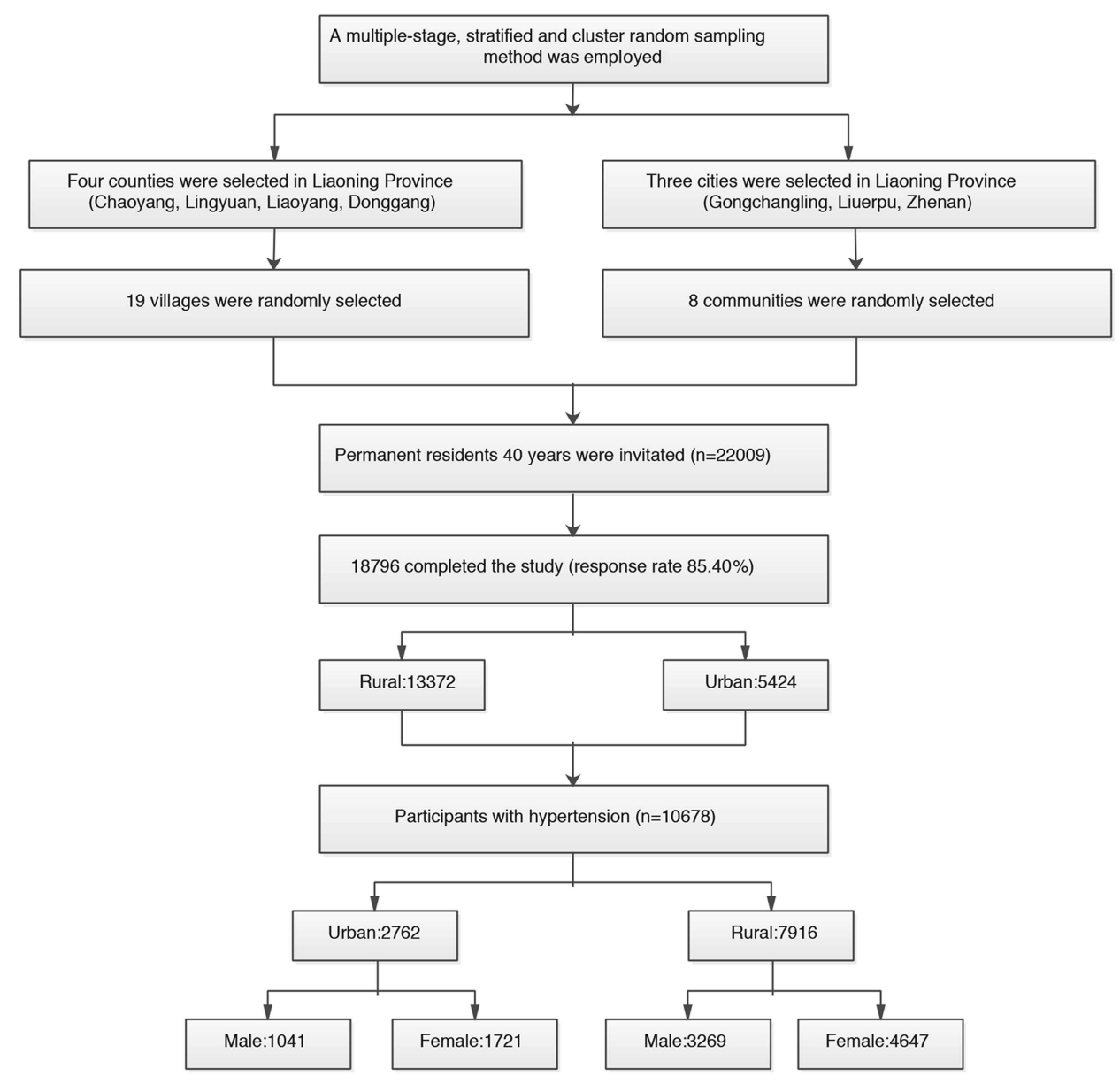

Figure I Flow chart of population selection.

was defined as hypertensive patients not meeting the criteria of hypertension anymore.

Dyslipidemia was determined according to the criteria of National Cholesterol Education Program-Third Adult Treatment Panel (ATP III). ${ }^{19}$ High LDL-C was defined as LDL-C $\geq 4.16 \mathrm{mmol} / \mathrm{L}$, low HDL-C was defined as HDL-C $<1.03 \mathrm{mmol} / \mathrm{L}$, high TG was defined as $\mathrm{TG} \geq 2.26 \mathrm{mmol} / \mathrm{L}$, high TC was defined as TC $\geq 6.21 \mathrm{mmol} / \mathrm{L}$. Diabetics was defined according to the World Health Organization (WHO) criteria: $\mathrm{FBG} \geq 7.0 \mathrm{mmol} / \mathrm{L}$ or $\mathrm{HbAlc} \geq 6.5 \%$ and/or self-reported diagnosis of diabetics that was identified by a certified physician previously. ${ }^{20}$

Current smoking ( $\geq 1$ cigarette/day and continued for $\geq 1$ year) and current drinking (any dose of alcohol, $\geq 1$ time/week) were determined according to the self-report. ${ }^{21}$ Lack of exercise was defined as failing to meet the standards for regular exercise including moderate-intensity exercise (equivalent to walking) for $\geq 30$ mins and $\geq 3$ times per week. Obese was defined as body mass index (BMI) $\geq 30 \mathrm{~kg} / \mathrm{m}^{22}$

\section{Statistical methods}

Descriptive statistics were calculated for all variables. Continuous variables with normal distribution are reported as means and standard deviations. Differences between groups were compared using a Chi-square test for categorical variables. The Student' $t$-test was used to compare differences in continuous variables between area and sex subgroups. Logistic regression was applied for risk factor analysis in urban and rural areas. Corresponding 95\% CIs were estimated for the parameters. All statistical analyses were conducted using SPSS22.0 (SPSS Inc., Chicago, IL, USA); $P$-values $<0.05$ were considered statistically significant.

\section{Results}

\section{Characteristics of the study population}

A total of 18,796 participants $(39.0 \%$ men and $61.0 \%$ women; $28.9 \%$ urban and $71.1 \%$ rural) completed the survey and were included in the analysis (Figure 1 and Table 1). The mean age of the population was $60.4 \pm 9.9$ 
Table I Characteristics of the study participants

\begin{tabular}{|c|c|c|c|c|c|c|c|}
\hline \multirow[t]{2}{*}{ Characteristics } & \multicolumn{2}{|l|}{ Region } & \multicolumn{2}{|l|}{ Sex } & \multirow[t]{2}{*}{ Total } & \multirow{2}{*}{$\begin{array}{l}P \text {-value } \\
\text { for region }\end{array}$} & \multirow{2}{*}{$\begin{array}{l}P \text {-value } \\
\text { for sex }\end{array}$} \\
\hline & Urban & Rural & Men & Women & & & \\
\hline Participants, n (\%) & $5424(28.9)$ & I3372 (7I.I) & $7336(39.0)$ & $11460(61.0)$ & $18796(100.0)$ & & \\
\hline Mean age, year & $60.9 \pm 9.5$ & $60.2 \pm 10.1$ & $61.3 \pm 10.2$ & $59.7 \pm 9.7$ & $60.4 \pm 9.9$ & $<0.001$ & $<0.001$ \\
\hline $40-49$ & 12.2 & 16.7 & 14.3 & 16.1 & 15.4 & $<0.001$ & $<0.001$ \\
\hline $50-59$ & 31.7 & 30.3 & 27.5 & 32.7 & 30.7 & & \\
\hline $60-69$ & 39.0 & 34.8 & 37.4 & 35.1 & 36.0 & & \\
\hline 70-79 & 14.5 & 15.3 & 17.2 & 13.7 & 15.1 & & \\
\hline$\geq 80$ & 2.6 & 3.0 & 3.6 & 2.4 & 2.9 & & \\
\hline \multicolumn{8}{|l|}{ Education, n (\%) } \\
\hline Primary school or lower & 25.1 & 59.3 & 42.3 & 54.0 & 49.4 & $<0.001$ & $<0.001$ \\
\hline Middle school & 53.0 & 32.4 & 42.6 & 35.6 & 38.3 & & \\
\hline High school or above & 21.9 & 8.3 & 15.1 & 10.4 & 12.2 & & \\
\hline \multicolumn{8}{|l|}{ Income, n (\%) } \\
\hline$<5000$ & 8.0 & 44.1 & 31.1 & 35.4 & 33.7 & $<0.001$ & $<0.001$ \\
\hline $5000-9999$ & 6.4 & 22.1 & 18.0 & 17.3 & 17.6 & & \\
\hline $10000-19999$ & 16.5 & 17.6 & 16.2 & 18.0 & 17.3 & & \\
\hline$\geq 20000$ & 69.0 & 16.1 & 34.6 & 29.3 & 31.4 & & \\
\hline Mean BMI, kg/m² & $24.9 \pm 3.4$ & $24.6 \pm 3.7$ & $24.4 \pm 3.6$ & $24.9 \pm 3.7$ & $24.7 \pm 3.7$ & $<0.001$ & $<0.001$ \\
\hline$<18.5$ & 1.6 & 3.5 & 3.3 & 2.7 & 2.9 & $<0.001$ & $<0.001$ \\
\hline $18.5-23.9$ & 39.9 & 42.1 & 44.6 & 39.4 & 41.5 & & \\
\hline $24.0-27.9$ & 41.9 & 37.2 & 37.7 & 39.2 & 38.6 & & \\
\hline$\geq 28.0$ & 16.6 & 17.2 & 14.4 & 18.7 & 17 & & \\
\hline Mean SBP, mmHg & $137.6 \pm 19.9$ & $|45| \pm 23.3$. & $|43.7 \pm 2| .5$ & $142.4 \pm 23.3$ & $142.9 \pm 22.6$ & $<0.001$ & $<0.001$ \\
\hline Mean DBP, mmHg & $83.4 \pm 10.6$ & $86.2 \pm 11.9$ & $87.1 \pm I I .5$ & $84.3 \pm 11.5$ & $85.4 \pm 11.6$ & $<0.001$ & $<0.001$ \\
\hline Current smoking, \% & 19.0 & 27.0 & 52.9 & 6.6 & 24.7 & $<0.001$ & $<0.001$ \\
\hline Current drinking, $\%$ & 20.4 & 28.9 & 53.4 & 9.1 & 26.4 & $<0.001$ & $<0.001$ \\
\hline Lack of exercise, \% & 8.9 & 15.1 & 12.0 & 14.1 & 13.3 & $<0.001$ & $<0.001$ \\
\hline $\begin{array}{l}\text { Family history of } \\
\text { hypertension, \% }\end{array}$ & 30.1 & 33.8 & 27.7 & 36.0 & 32.8 & $<0.001$ & $<0.001$ \\
\hline Diabetes & 20.2 & 15.8 & 16.5 & 17.4 & 17.1 & $<0.001$ & 0.113 \\
\hline High TC & 14.6 & 14.1 & 10.2 & 16.8 & 14.2 & 0.298 & $<0.001$ \\
\hline High TG & 19.9 & 16.8 & 16.2 & 18.6 & 17.7 & $<0.001$ & $<0.001$ \\
\hline Low HDL & 28.4 & 4.5 & 13.0 & 10.4 & 11.4 & $<0.001$ & $<0.001$ \\
\hline High LDL & 9.3 & 4.2 & 3.7 & 6.9 & 5.7 & $<0.001$ & $<0.001$ \\
\hline
\end{tabular}

Abbreviations: BMI, body mass index; SBP, systolic blood pressure; DBP, diastolic blood pressure; TC, total cholesterol; TG, triglyceride; HDL, high-density lipoprotein; LDL, low-density lipoprotein.

years $(60.9 \pm 9.5$ years in urban and $60.2 \pm 10.1$ years in rural). A significant difference was observed between urban and rural for all characteristics including age, education level, income, BMI, prevalence of current smoking, current drinking, lack of exercise, and family history of hypertension. Rural residents had a higher prevalence of low education, low income, low BMI, current smoking, current drinking, lack of exercise, and family history of hypertension than did urban residents (Table 1). 


\section{Prevalence and classification of hypertension}

Among adults aged $\geq 40$ years in northeast China, the overall crude and age-standardized prevalence of hypertension was $56.8 \%$ (urban $50.9 \%$ and rural $59.2 \%$, $p<0.001$; men $58.8 \%$ and women $55.6 \%, p<0.001)$ and $50.2 \%$ (urban $43.5 \%$ and rural $52.5 \%$; men $53.7 \%$ and women $48.6 \%$ ). The overall crude and age-standardized prevalence of prehypertension was $31.5 \%$ (urban $34.9 \%$ and rural $30.0 \%, p<0.001$, men $32.6 \%$ and women $30.9 \%$, $p=0.011$ ) and $34.3 \%$ (urban $37.6 \%$ and rural $33.1 \%$, men $35.8 \%$ and women $33.2 \%$ ), respectively. Moreover, there were significant differences in crude prevalence of hypertension and prehypertension for each subgroup $(p<0.05)$.

The age-standardized prevalence of hypertension and prehypertension were higher in males than in females (58.8\% vs $55.6 \%$ and $32.6 \%$ vs $30.9 \%$, respectively). Compared with the urban population, although the prevalence of prehypertension was lower in the rural population, the prevalence of stage 2 and stage 3 hypertension was significantly higher $(18.3 \%$ vs 11.1 , and $9.5 \%$ vs 4.2 , respectively) (Table 2 and Figure 2).

\section{Levels of SBP and DBP}

The mean SBP and DBP were $142.9 \pm 22.6 \mathrm{mmHg}$ and 85.4 $\pm 11.6 \mathrm{mmHg}$ in the overall population (Table 1 and Figure 3 ). The mean SBP and DBP were significantly higher in rural areas compared with those in urban areas $(145.1 \pm 23.3 \mathrm{mmHg}$ vs $137.6 \pm 19.9 \mathrm{mmHg}$, and $86.2 \pm 11.9 \mathrm{mmHg}$ vs $83.4 \pm 10.6$ $\mathrm{mmHg}$, respectively, $p<0.001$ ).

Moreover, in addition to significant differences across all age groups, the mean SBP and DBP were more likely to be higher in male participants with a low-education level, low income, high BMI, family history of hypertension, and comorbidities including diabetes and dyslipidemia $(p<0.05)$ (Table 2).

\section{Awareness, treatment, and control of hypertension}

Among those with hypertension, $48.5 \%$ were aware of their diagnosis; $38.0 \%$ were taking antihypertensive medications, but only $5.7 \%$ achieved control of blood pressure. Among those who received medical treatment, only $14.9 \%$ achieved control of blood pressure. The rates of awareness, treatment, and control are shown in Table 3 and Figure 4. The awareness, treatment, and control rates of hypertension in urban areas were all significantly higher than those in rural areas $(52.5 \%$ vs $47.0 \%, 46.9 \%$ vs $34.9 \%$, and $11.4 \%$ vs $3.7 \%$, respectively, $p<0.001$ ).

\section{Related risk factors in the hypertensive population}

Significant differences were found between urban and rural areas among the risk factors analyzed for both prevalence and control of hypertension. Multivariate regression analysis identified that male gender, older age, and family history of hypertension, diabetes, and dyslipidemia were common risk factors for prevalent hypertension in both urban and rural areas. What is noteworthy is that lack of exercise (OR, 1.24; 95\% CI, 1.10-1.38) was associated with an increased risk of hypertension in rural populations, and overweight/obesity (OR, 2.01; 95\% CI, 1.79-2.27) and current drinking (OR, 1.20; 95\% CI, 1.01-1.41) were associated with increased risk of hypertension in urban areas $(p<0.05)$.

Participants with a high education level were likely to have low prevalence of hypertension for both urban and rural areas; however, higher household income $(>20,000$ yuan/year) (OR, $0.78 ; 95 \% \mathrm{CI}, 0.69-0.88)$ was shown to be a protective factor against hypertension only in rural areas $(p<0.05)$ (Table 6). Moreover, compared with men, women who lacked exercise (OR, 1.45; 95\% CI, 1.081.94) were more likely to have hypertension.

According to the results of the multivariate regression model, men were unlikely to control their hypertension at normal levels (OR, 0.49 ; 95\% CI, 0.36-0.67 in urban and OR, $0.65 ; 95 \% \mathrm{CI}, 0.51-0.85$ in rural) compared to women. Moreover, people with a family history of hypertension and dyslipidemia were more likely to have higher control rate of hypertension both for urban and rural areas. Furthermore, hypertensive people with younger age, lack of exercise, and diabetes in rural areas, and participants with a higher level of education and current smoking in urban areas tended to have a higher control rate. Compared with men, hypertensive women with current smoking and diabetes were likely to achieve normal blood pressure (Tables 4 and 5).

\section{Classification based on the $2017 \mathrm{ACC} /$ AHA guidelines}

Based on the 2017 ACC/AHA guidelines, the prevalence of hypertension was $80.6 \%$, and the control rate was only $0.9 \%$. Even among those participants who received medical treatment, only $3.3 \%$ became normotensive (Figure 5 and Table 6 ). 
Table 2 Blood pressure level and prevalence of prehypertension, and hypertension by characteristics

\begin{tabular}{|c|c|c|c|c|c|c|c|c|c|}
\hline \multirow[t]{2}{*}{ Characteristics } & \multirow[t]{2}{*}{$\mathbf{N}$} & \multicolumn{2}{|c|}{ Blood pressure $(\mathrm{mmHg})$} & \multicolumn{5}{|c|}{ Hypertension classification (\%) } & \multirow{2}{*}{$\begin{array}{l}\text { Hypertension } \\
\text { prevalence (\%) }\end{array}$} \\
\hline & & SBP & DBP & Normal & $\begin{array}{l}\text { Prehyper- } \\
\text { tension }\end{array}$ & $\begin{array}{l}\text { Stage } \\
\text { I }\end{array}$ & $\begin{array}{l}\text { Stage } \\
2\end{array}$ & $\begin{array}{l}\text { Stage } \\
3\end{array}$ & \\
\hline Overall & 18,796 & $142.9 \pm 22.6$ & $85.4 \pm 11.6$ & 14.9 & 31.5 & 29.4 & 16.2 & 8.0 & 56.8 \\
\hline $\begin{array}{l}\text { Age group } \\
\begin{array}{l}40-49 \\
50-59 \\
60-69 \\
70-79 \\
\geq 80 \\
\text { P-value }\end{array}\end{array}$ & $\begin{array}{l}2895 \\
5764 \\
6763 \\
2830 \\
544\end{array}$ & $\begin{array}{l}131.3 \pm 19.1 \\
139.0 \pm 20.7 \\
146.1 \pm 22.2 \\
152.9 \pm 23.4 \\
154.0 \pm 24.3 \\
<0.00 \mid\end{array}$ & $\begin{array}{l}84.5 \pm 11.9 \\
86.0 \pm 11.6 \\
85.7 \pm 11.4 \\
84.5 \pm 11.5 \\
84.2 \pm 12.3 \\
<0.001\end{array}$ & $\begin{array}{l}24.8 \\
16.8 \\
12.0 \\
8.9 \\
8.1\end{array}$ & $\begin{array}{l}39.5 \\
36.5 \\
29.4 \\
20.9 \\
19.7 \\
<0.001\end{array}$ & $\begin{array}{l}23.0 \\
27.7 \\
31.7 \\
33.0 \\
34.4\end{array}$ & $\begin{array}{l}8.9 \\
13.3 \\
18.2 \\
23.6 \\
22.8\end{array}$ & $\begin{array}{l}3.7 \\
5.8 \\
8.8 \\
13.5 \\
15.1\end{array}$ & $\begin{array}{l}36.9 \\
50.0 \\
62.5 \\
73.9 \\
75.4 \\
<0.001\end{array}$ \\
\hline $\begin{array}{l}\text { Sex } \\
\text { Male } \\
\text { Female } \\
\text { P-value }\end{array}$ & $\begin{array}{l}7336 \\
11,460\end{array}$ & $\begin{array}{l}|43.7 \pm 2| .5 \\
\mid 42.4 \pm 23.3 \\
<0.00 \mid\end{array}$ & $\begin{array}{l}87.1 \pm 11.5 \\
84.311 .5 \\
<0.001\end{array}$ & $\begin{array}{l}11.0 \\
17.3\end{array}$ & $\begin{array}{l}32.6 \\
30.9 \\
0.011\end{array}$ & $\begin{array}{l}31.3 \\
28.2\end{array}$ & $\begin{array}{l}17.2 \\
15.5\end{array}$ & $\begin{array}{l}7.9 \\
8.0\end{array}$ & $\begin{array}{l}58.8 \\
55.6 \\
<0.001\end{array}$ \\
\hline $\begin{array}{l}\text { Education, } \mathbf{n} \text { (\%) } \\
\text { Primary school or lower } \\
\text { Middle school } \\
\text { High school or above } \\
\text { P-value }\end{array}$ & $\begin{array}{l}9289 \\
7205 \\
2302\end{array}$ & $\begin{array}{l}146.9 \pm 23.6 \\
139.6 \pm 21.0 \\
137.0 \pm 20.2 \\
<0.001\end{array}$ & $\begin{array}{l}85.8 \pm 12.0 \\
85.0 \pm 11.2 \\
84.9 \pm 11.0 \\
<0.001\end{array}$ & $\begin{array}{l}12.5 \\
16.5 \\
19.3\end{array}$ & $\begin{array}{l}27.2 \\
35.9 \\
35.4 \\
<0.001\end{array}$ & $\begin{array}{l}30.6 \\
28.3 \\
28.4\end{array}$ & $\begin{array}{l}19.1 \\
13.5 \\
12.9\end{array}$ & $\begin{array}{l}10.7 \\
5.8 \\
4.0\end{array}$ & $\begin{array}{l}63.2 \\
50.9 \\
49.5 \\
<0.001\end{array}$ \\
\hline $\begin{array}{l}\text { Income, } \mathbf{n} \text { (\%) } \\
<5000 \\
5000-9999 \\
10000-19999 \\
\geq 20000 \\
\text { P-value }\end{array}$ & $\begin{array}{l}6336 \\
3303 \\
3257 \\
5900\end{array}$ & $\begin{array}{l}|48.7 \pm 24 .| \\
\mid 43.2 \pm 22.4 \\
|40.3 \pm 2| .4 \\
\mid 37.9 \pm 20.3 \\
<0.00 \mid\end{array}$ & $\begin{array}{l}85.9 \pm 12.2 \\
85.8 \pm 11.5 \\
85.3 \pm 11.5 \\
84.6 \pm 11.0 \\
<0.001\end{array}$ & $\begin{array}{l}11.2 \\
14.4 \\
16.1 \\
18.3\end{array}$ & $\begin{array}{l}26.5 \\
30.5 \\
34.2 \\
36.1 \\
<0.001\end{array}$ & $\begin{array}{l}30.0 \\
30.0 \\
28.6 \\
28.9\end{array}$ & $\begin{array}{l}20.3 \\
17.2 \\
14.6 \\
12.1\end{array}$ & $\begin{array}{l}12.0 \\
7.9 \\
6.5 \\
4.6\end{array}$ & $\begin{array}{l}64.9 \\
57.6 \\
52.7 \\
50.0 \\
<0.001\end{array}$ \\
\hline 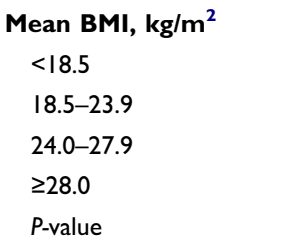 & $\begin{array}{l}549 \\
7790 \\
7253 \\
3200\end{array}$ & $\begin{array}{l}138.2 \pm 23.6 \\
138.9 \pm 22.2 \\
144.2 \pm 22.0 \\
150.4 \pm 22.6 \\
<0.00 \mid\end{array}$ & $\begin{array}{l}81.4 \pm 11.5 \\
82.9 \pm 11.1 \\
86.2 \pm 11.1 \\
90.3 \pm 11.9 \\
<0.001\end{array}$ & $\begin{array}{l}21.9 \\
19.0 \\
12.8 \\
8.2\end{array}$ & $\begin{array}{l}33.7 \\
35.5 \\
31.0 \\
22.9 \\
<0.001\end{array}$ & $\begin{array}{l}25.3 \\
26.6 \\
30.8 \\
33.9\end{array}$ & $\begin{array}{l}13.5 \\
13.2 \\
17.0 \\
22.2\end{array}$ & $\begin{array}{l}5.6 \\
5.7 \\
8.4 \\
12.8\end{array}$ & $\begin{array}{l}46.6 \\
47.9 \\
60.1 \\
72.9 \\
<0.001\end{array}$ \\
\hline $\begin{array}{l}\text { Current smoking, \% } \\
\text { Yes } \\
\text { No } \\
\text { P-value }\end{array}$ & $\begin{array}{l}4636 \\
|4,| 40\end{array}$ & $\begin{array}{l}|4| .7 \pm 22 . \mid \\
\mid 43.3 \pm 22.8 \\
<0.00 \mid\end{array}$ & $\begin{array}{l}86.0 \pm 11.8 \\
85.2 \pm 11.5 \\
<0.001\end{array}$ & $\begin{array}{l}14.7 \\
14.9\end{array}$ & $\begin{array}{l}34.0 \\
30.7 \\
<0.001\end{array}$ & $\begin{array}{l}28.5 \\
29.7\end{array}$ & $\begin{array}{l}15.1 \\
16.6\end{array}$ & $\begin{array}{l}7.7 \\
8.1\end{array}$ & $\begin{array}{l}53.9 \\
57.7 \\
<0.001\end{array}$ \\
\hline $\begin{array}{l}\text { Current drinking, \% } \\
\text { Yes } \\
\text { No } \\
P \text {-value } \\
\text { Lack of exercise, \% } \\
\text { Yes } \\
\text { No } \\
P \text {-value }\end{array}$ & $\begin{array}{l}2500 \\
16,296\end{array}$ & $\begin{array}{l}142.9 \pm 21.7 \\
142.9 \pm 22.9 \\
0.934 \\
\mid 48.7 \pm 24.7 \\
\mid 42.0 \pm 22.1 \\
<0.00 \mid\end{array}$ & $\begin{array}{l}87.2 \pm 11.6 \\
84.7 \pm 11.5 \\
<0.001 \\
86.6 \pm 12.4 \\
85.2 \pm 11.4 \\
<0.001\end{array}$ & $\begin{array}{l}12.5 \\
15.2\end{array}$ & $\begin{array}{l}33.0 \\
31.0 \\
0.012 \\
\\
24.5 \\
32.6 \\
<0.001\end{array}$ & $\begin{array}{l}30.1 \\
29.2 \\
\\
\\
29.9 \\
29.3\end{array}$ & $\begin{array}{l}16.8 \\
16.0 \\
\\
20.4 \\
15.5\end{array}$ & $\begin{array}{l}7.9 \\
8.0 \\
\\
12.7 \\
7.3\end{array}$ & $\begin{array}{l}56.9 \\
56.8 \\
0.842 \\
\\
66.8 \\
55.3 \\
<0.001\end{array}$ \\
\hline Family history of hypert & sion, \% & & & & & & & & \\
\hline $\begin{array}{l}\text { Yes } \\
\text { No } \\
P \text {-value }\end{array}$ & $\begin{array}{l}6159 \\
12,637\end{array}$ & $\begin{array}{l}\mid 46.9 \pm 23.4 \\
\mid 40.9 \pm 22.0 \\
<0.00 \mid\end{array}$ & $\begin{array}{l}88.0 \pm 11.6 \\
84.1 \pm 11.1 \\
<0.001\end{array}$ & $\begin{array}{l}13.5 \\
15.6\end{array}$ & $\begin{array}{l}25.1 \\
34.7 \\
<0.001\end{array}$ & $\begin{array}{l}30.2 \\
29.0\end{array}$ & $\begin{array}{l}20.1 \\
14.3\end{array}$ & $\begin{array}{l}11.1 \\
6.4\end{array}$ & $\begin{array}{l}66.5 \\
52.1 \\
<0.001\end{array}$ \\
\hline
\end{tabular}

(Continued) 
Table 2 (Continued).

\begin{tabular}{|c|c|c|c|c|c|c|c|c|c|}
\hline \multirow[t]{2}{*}{ Characteristics } & \multirow[t]{2}{*}{$\mathbf{N}$} & \multicolumn{2}{|c|}{ Blood pressure $(\mathrm{mmHg})$} & \multicolumn{5}{|c|}{ Hypertension classification (\%) } & \multirow{2}{*}{$\begin{array}{l}\text { Hypertension } \\
\text { prevalence (\%) }\end{array}$} \\
\hline & & SBP & DBP & Normal & $\begin{array}{l}\text { Prehyper- } \\
\text { tension }\end{array}$ & $\begin{array}{l}\text { Stage } \\
\text { I }\end{array}$ & $\begin{array}{l}\text { Stage } \\
2\end{array}$ & $\begin{array}{l}\text { Stage } \\
3\end{array}$ & \\
\hline \multicolumn{10}{|l|}{ Region } \\
\hline Urban & 5424 & $137.6 \pm 19.9$ & $83.4 \pm 10.6$ & 20.0 & 34.9 & 29.8 & 11.1 & 4.2 & 50.9 \\
\hline Rural & 13,372 & $\mid 45.1 \pm 23.3$ & $86.2 \pm 11.9$ & 12.8 & 30.2 & 29.2 & 18.3 & 9.5 & 59.2 \\
\hline$P$-value & & $<0.001$ & $<0.001$ & & $<0.001$ & & & & $<0.001$ \\
\hline \multicolumn{10}{|l|}{ Diabetes, \% } \\
\hline Yes & 3200 & $150.4 \pm 22.9$ & $86.9 \pm 11.9$ & 10.6 & 23.0 & 32.7 & 21.8 & 11.9 & 71.7 \\
\hline No & 15,560 & $|4| .3 \pm 22.3$ & $85.1 \pm 11.5$ & 15.8 & 33.3 & 28.7 & 15 & 7.2 & 53.7 \\
\hline$P$-value & & $<0.001$ & $<0.001$ & & $<0.001$ & & & & $<0.001$ \\
\hline \multicolumn{10}{|l|}{ High TC, \% } \\
\hline Yes & 2670 & $148.4 \pm 23.3$ & $87.1 \pm 11.6$ & 10.2 & 26.9 & 31.3 & 19.7 & 11.9 & 66.2 \\
\hline No & 16,089 & $142.0 \pm 22.4$ & $85.1 \pm 11.6$ & 15.7 & 32.3 & 29.1 & 15.6 & 7.3 & 55.2 \\
\hline$P$-value & & $<0.001$ & $<0.001$ & & $<0.001$ & & & & $<0.001$ \\
\hline \multicolumn{10}{|l|}{ High TG, \% } \\
\hline Yes & 3316 & $147.3 \pm 22.5$ & $88.2 \pm 11.4$ & 11.0 & 26.0 & 32.4 & 19.9 & 10.8 & 67.6 \\
\hline No & 15,443 & $141.9 \pm 22.5$ & $84.8 \pm 11.5$ & 15.7 & 32.8 & 28.8 & 15.4 & 7.4 & 54.5 \\
\hline$P$-value & & $<0.001$ & $<0.001$ & & $<0.001$ & & & & $<0.001$ \\
\hline \multicolumn{10}{|l|}{ Low HDL, \% } \\
\hline Yes & $2|4|$ & $142.5 \pm 20.5$ & $84.7 \pm 11.4$ & 14.5 & 32.7 & 31.1 & 15.4 & 6.4 & 57.4 \\
\hline No & 16,618 & $142.9 \pm 22.9$ & $85.5 \pm 11.6$ & 14.9 & 31.4 & 29.2 & 16.3 & 8.2 & 56.7 \\
\hline$P$-value & & 0.380 & 0.003 & & 0.229 & & & & 0.512 \\
\hline \multicolumn{10}{|l|}{ High LDL, \% } \\
\hline Yes & 1064 & $148.4 \pm 24.2$ & $86.3 \pm 11.5$ & 10.6 & 27.2 & 30.2 & 19.4 & 12.7 & 65.3 \\
\hline No & 17,695 & $142.5 \pm 22.5$ & $85.3 \pm 11.6$ & 15.1 & 31.8 & 29.4 & 16.0 & 7.7 & 56.3 \\
\hline$P$-value & & $<0.001$ & 0.011 & & 0.001 & & & & 0.001 \\
\hline
\end{tabular}

Abbreviations: BMI, body mass index; SBP, systolic blood pressure; DBP, diastolic blood pressure; TC, total cholesterol; TG, triglyceride; HDL, high-density lipoprotein; LDL, low-density lipoprotein.

Compared to urban areas, the prevalence of hypertension (76.6\% vs $82.2 \%$ ) was higher in rural areas; however, the control rate remains unacceptably low (1.8\% vs $0.6 \%)$. Among those who received medical treatment, only 5.6\% and $2.2 \%$ achieved control of blood pressure in urban and rural regions, respectively, according to the 2017 ACC/AHA guidelines.

Although the 2017 ACC/AHA guidelines resulted in a substantial increase in the prevalence of hypertension both in rural and urban areas, the increase rates were higher in urban areas than in rural areas $(27.9 \%$ vs $25.7 \%, p<0.05)$. Males had a higher change than females $(28.3 \%$ vs $25.1 \%$ ). Moreover, the difference in control rate was $9.7 \%$ in urban areas and $3.1 \%$ in rural areas. Furthermore, females had a more significant change than males (5.6\% vs 3.5\%). In addition, the 2017 ACC/AHA guidelines led to a substantial change in people aged 60 to
80 years compared with the Chinese guidelines, especially in urban areas.

\section{Discussion}

The present study provides a comprehensive estimation on the disparities of current hypertension status as well as related risk factors in a representative sample for urban and rural areas in northeast China. In addition to the direct comparisons of the status and risk factor profile according to the Chinese guidelines, we estimated the burden of hypertension in those areas under the 2017 ACC/AHA High Blood Pressure Guidelines. The key findings from the population-based cross-sectional survey were as follows: (1) high prevalence of hypertension and prehypertension (50.2\% and $34.3 \%$, respectively) but low awareness, treatment, and blood pressure control $(48.5 \%$, $38.0 \%$, and $5.7 \%$ ) among hypertensive individuals represent a considerable cardiovascular disease risk burden in northeast 

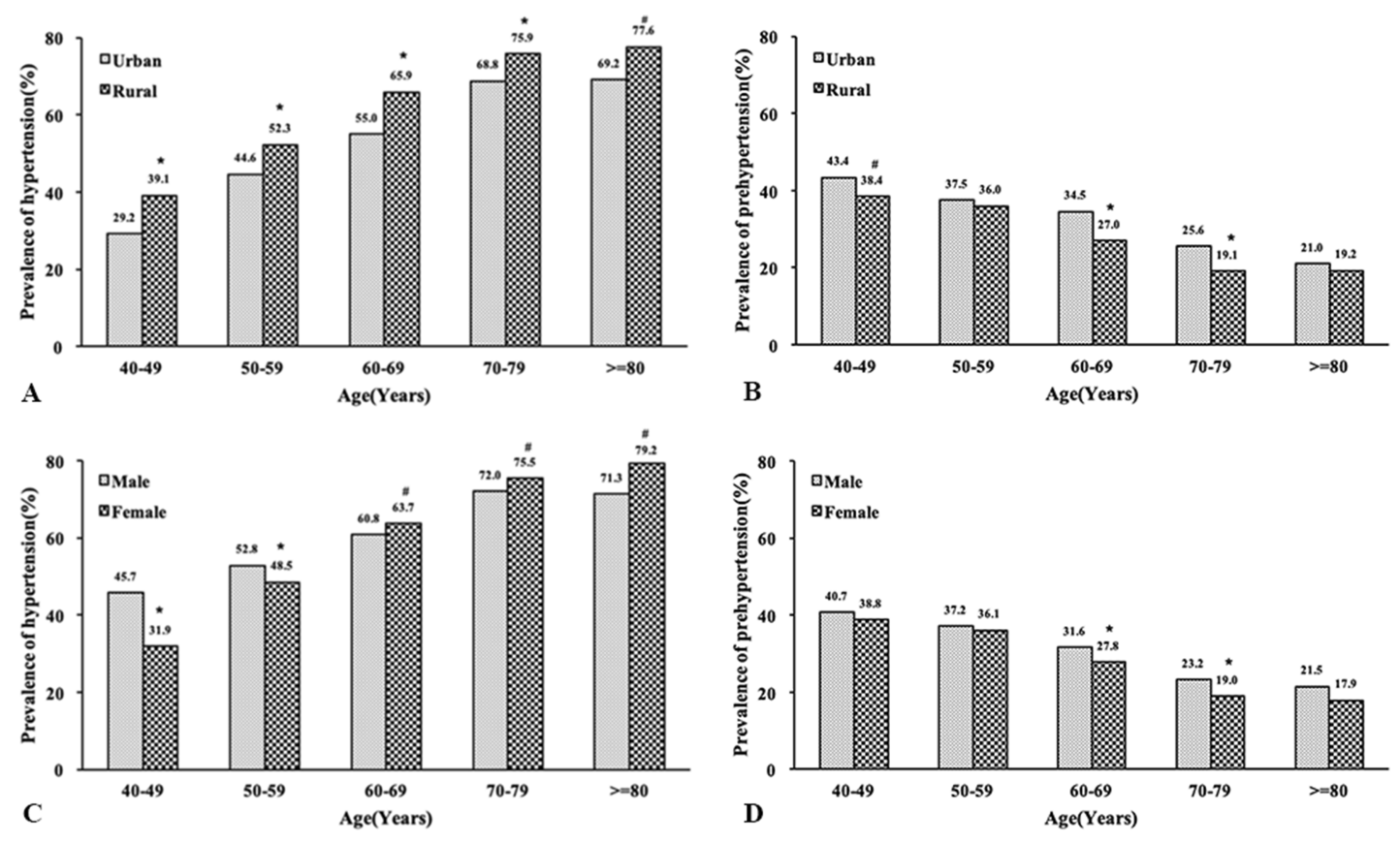

Figure 2 Prevalence of hypertension and prehypertension per 100 (with $95 \% \mathrm{Cl}$ ) among urban and rural adults in northeast China by region and sex. (A) Prevalence of hypertension by region, (B) prevalence of prehypertension by region, (C) prevalence of hypertension by sex, (D) prevalence of prehypertension by sex.
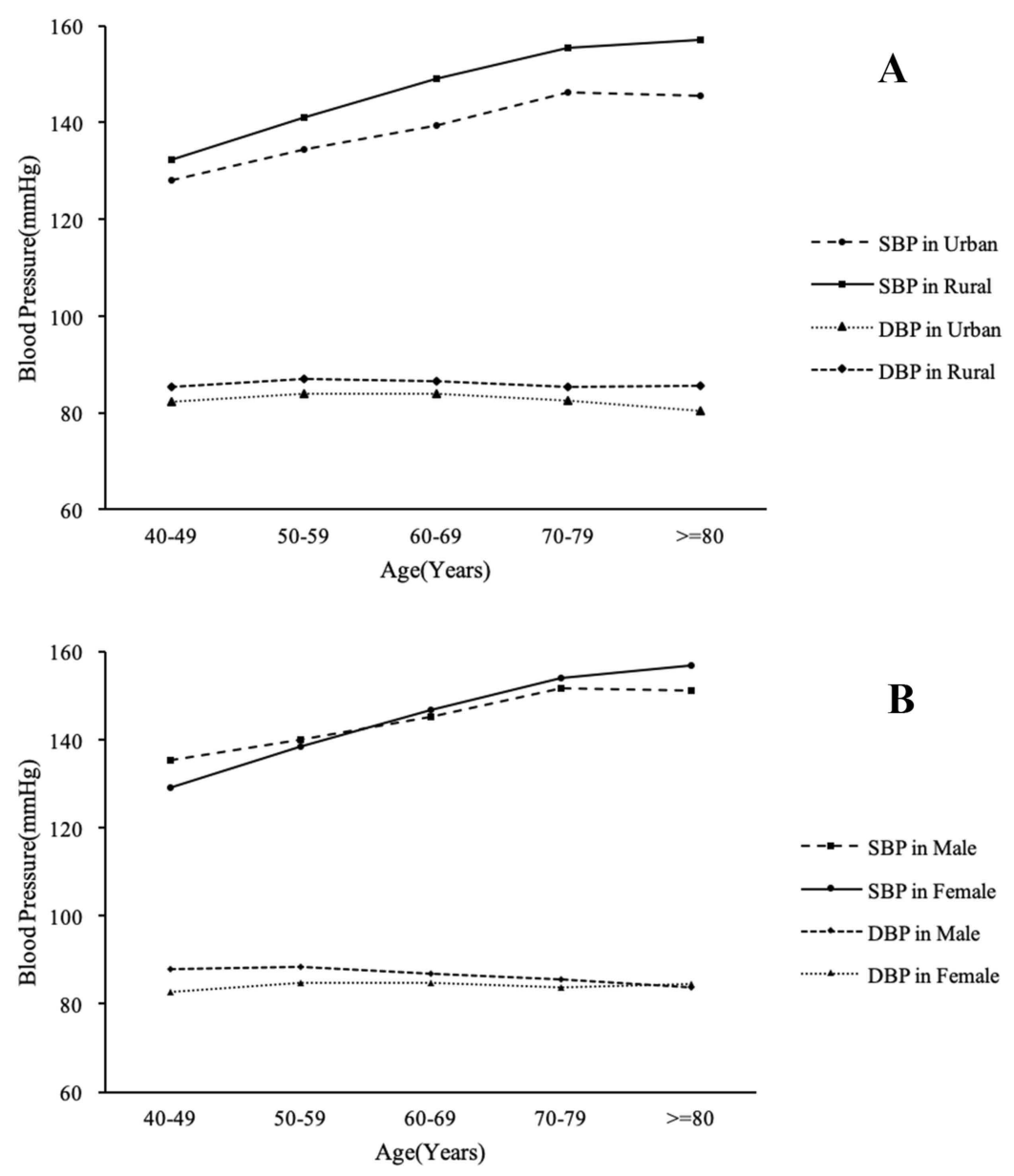

Figure 3 Levels of systolic blood pressure and diastolic blood pressure in adults in urban and rural northeast China by region and sex. (A) By region, (B) by sex. 
Table 3 Awareness, treatment, and control rates of hypertension by characteristics

\begin{tabular}{|c|c|c|c|c|}
\hline Characteristics & Awareness & Treatment & Control & Control among treated patients \\
\hline Overall & 48.5 & 38.0 & 5.7 & 14.9 \\
\hline \multicolumn{5}{|l|}{ Age group } \\
\hline $40-49$ & 37.4 & 24.7 & 3.3 & 13.3 \\
\hline $50-59$ & 46.3 & 36.0 & 6.5 & 18.0 \\
\hline $60-69$ & 50.4 & 41.2 & 6.2 & 15.0 \\
\hline $70-79$ & 53.1 & 42.2 & 5.1 & 12.0 \\
\hline$\geq 80$ & 48.0 & 31.7 & 4.1 & 13.1 \\
\hline$P$-value & $<0.001$ & $<0.001$ & $<0.001$ & 0.005 \\
\hline \multicolumn{5}{|l|}{ Sex } \\
\hline Male & 41.6 & 29.9 & 4.1 & 15.6 \\
\hline Female & 53.1 & 43.5 & 6.8 & 13.6 \\
\hline$P$-value & $<0.001$ & $<0.001$ & $<0.001$ & 0.102 \\
\hline \multicolumn{5}{|l|}{ Education, n (\%) } \\
\hline Primary school or lower & 49.0 & 37.7 & 4.5 & 12.0 \\
\hline Middle school & 47.4 & 37.9 & 6.6 & 17.4 \\
\hline High school or above & 49.3 & 39.7 & 8.5 & 21.5 \\
\hline$P$-value & 0.293 & 0.455 & $<0.001$ & $<0.001$ \\
\hline \multicolumn{5}{|l|}{ Income, n (\%) } \\
\hline$<5000$ & 49.2 & 38.4 & 4.1 & 10.6 \\
\hline 5000-9999 & 48.4 & 35.2 & 4.4 & 12.5 \\
\hline $10000-19999$ & 46.6 & 35.8 & 5.7 & 16.0 \\
\hline$\geq 20000$ & 48.5 & 40.4 & 8.7 & 21.5 \\
\hline$P$-value & 0.357 & 0.001 & $<0.001$ & $<0.001$ \\
\hline \multicolumn{5}{|l|}{ Mean BMI, kg/m² } \\
\hline$<18.5$ & 37.5 & 23.8 & 4.7 & 19.7 \\
\hline $18.5-23.9$ & 42.1 & 30.8 & 4.9 & 16.0 \\
\hline $24.0-27.9$ & 49.6 & 39.9 & 6.4 & 16.1 \\
\hline$\geq 28.0$ & 57.6 & 47.5 & 5.6 & 11.7 \\
\hline$P$-value & $<0.001$ & $<0.001$ & 0.034 & 0.005 \\
\hline \multicolumn{5}{|l|}{ Current smoking, $\%$} \\
\hline Yes & 41.4 & 29.3 & 4.9 & 16.6 \\
\hline No & 50.6 & 40.6 & 5.9 & 14.6 \\
\hline$P$-value & $<0.001$ & $<0.001$ & 0.049 & 0.158 \\
\hline \multicolumn{5}{|l|}{ Current drinking, $\%$} \\
\hline Yes & 38.5 & 26.5 & 3.9 & 14.7 \\
\hline No & 52.1 & 42.1 & 6.3 & 15.0 \\
\hline$P$-value & $<0.001$ & $<0.001$ & $<0.001$ & 0.842 \\
\hline \multicolumn{5}{|l|}{ Lack of exercise, \% } \\
\hline Yes & 57.7 & 45.8 & 5.7 & 12.4 \\
\hline No & 46.8 & 36.5 & 5.7 & 15.5 \\
\hline$P$-value & $<0.001$ & $<0.001$ & 0.974 & 0.031 \\
\hline \multicolumn{5}{|c|}{ Family history of hypertension, \% } \\
\hline Yes & 62.6 & 51.7 & 7.6 & 14.8 \\
\hline No & 39.7 & 29.5 & 4.5 & 15.1 \\
\hline$P$-value & $<0.001$ & $<0.001$ & $<0.001$ & $0.78 \mathrm{I}$ \\
\hline
\end{tabular}

(Continued) 
Table 3 (Continued).

\begin{tabular}{|c|c|c|c|c|}
\hline Characteristics & Awareness & Treatment & Control & Control among treated patients \\
\hline \multicolumn{5}{|l|}{ Region } \\
\hline Urban & 52.5 & 46.9 & 11.4 & 24.4 \\
\hline Rural & 47.0 & 34.9 & 3.7 & 10.5 \\
\hline$P$-value & $<0.001$ & $<0.001$ & $<0.001$ & $<0.001$ \\
\hline \multicolumn{5}{|l|}{ Diabetes } \\
\hline Yes & 59.5 & 51.2 & 7.4 & 14.5 \\
\hline No & 45.4 & 34.4 & 5.2 & 15.2 \\
\hline$P$-value & $<0.001$ & $<0.001$ & $<0.001$ & 0.571 \\
\hline \multicolumn{5}{|l|}{ High TC } \\
\hline Yes & 47.5 & 39.5 & 5.0 & 12.6 \\
\hline No & 48.6 & 37.7 & 5.8 & 15.5 \\
\hline$P$-value & 0.37 & 0.146 & 0.159 & 0.053 \\
\hline \multicolumn{5}{|l|}{ High TG } \\
\hline Yes & 53.9 & 44.2 & 6.7 & I5.I \\
\hline No & 47 & 36.3 & 5.4 & 14.9 \\
\hline$P$-value & $<0.001$ & $<0.001$ & 0.02 & 0.875 \\
\hline \multicolumn{5}{|l|}{ Low HDL } \\
\hline Yes & 52.8 & 46.1 & 8.0 & 17.5 \\
\hline No & 47.9 & 36.9 & 5.4 & 14.6 \\
\hline$P$-value & 0.001 & $<0.001$ & $<0.001$ & 0.074 \\
\hline \multicolumn{5}{|l|}{ High LDL } \\
\hline Yes & 48.3 & 43.6 & 4.7 & 10.9 \\
\hline No & 48.5 & 37.6 & 5.8 & 15.3 \\
\hline$P$-value & 0.952 & 0.002 & 0.268 & 0.038 \\
\hline
\end{tabular}

Abbreviations: BMI, body mass index; TC, total cholesterol; TG, triglyceride; HDL, high-density lipoprotein; LDL, low-density lipoprotein.

China. (2) Significant urban-rural differences in status of hypertension as well as associated risk factors were observed. The prevalence of hypertension was higher, but the control rate was lower in rural residents compared with urban residents ( $52.5 \%$ vs $43.5 \%$ and $3.7 \%$ vs $11.4 \%$, respectively). In addition, the varied related risk factors for prevalence and control of hypertension between urban and rural areas indicate that different strategies are urgently needed in northeast China. (3) Under the 2017 ACC/AHA hypertension guidelines, the hypertension prevalence increased approximately two-thirds, especially in urban residents 60-80 years of age. However, the control rate decreased dramatically, especially in urban areas, suggesting the substantial risks and complications of hypertension in the coming decades. Therefore, fundamental structural changes are urgently needed to address hypertension in terms of reducing cardiovascular risk in northeast China. Moreover, since the majority of the Liaoning residents had low-to-middle income, our results are likely to be generally applicable to the population with same socioeconomic status in China.
Hypertension represents the leading cause of death, responsible for nearly 7.5 million premature deaths or $12.8 \%$ of all deaths worldwide. ${ }^{23}$ With the rapid economic progression, urbanization, and aging of the Chinese population, the epidemiology of hypertension is in a dynamic phase. ${ }^{24}$ Particularly in rural areas, urbanization is associated with potential lifestyle changes, which further lead to an increase in cardiovascular risk factors. Although a previous study showed convergence in urban-rural prevalence of hypertension at a national level, the geographic, demographic, and socioeconomic characteristics in different regions might differ widely. ${ }^{7}$ Moreover, northeast China bears a significant proportion of the national burden of morbidity and mortality due to hypertension; effective prevention and management of hypertension could reduce a large percentage of cardiovascular diseases in China. Our study indicated the steep increase in prevalence of hypertension, and stage 2 and above hypertension. High mean blood pressure levels in addition to frustratingly low 

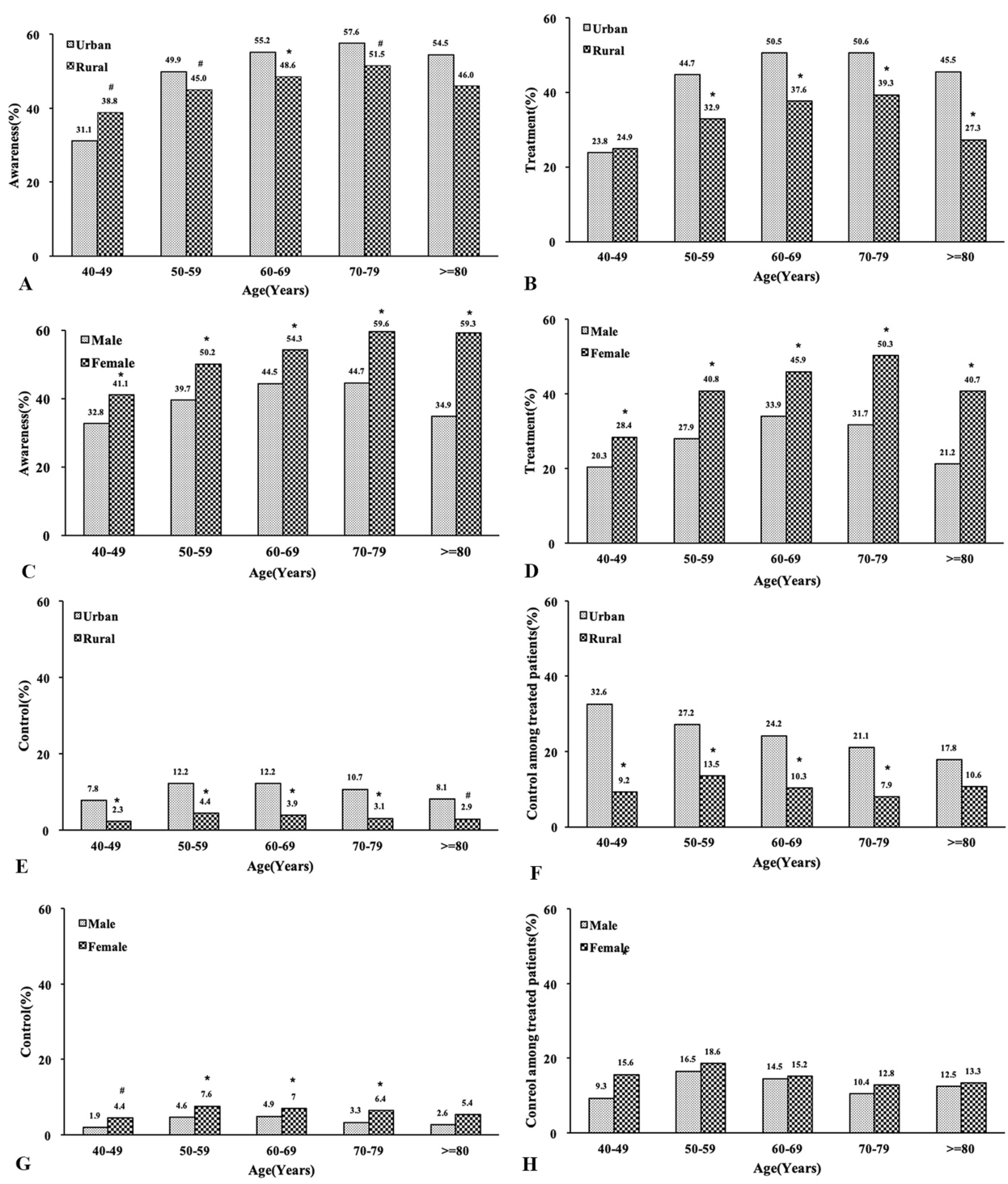

Figure 4 Awareness, treatment, and control rates among adults $\geq 40$ years of northeast China by region. (A) Awareness rate by region, (B) treatment rate by region, $(\mathbf{C})$ awareness rate by sex, (D) treatment rate by sex, $(\mathbf{E})$ control rate by region, $(\mathbf{F})$ control rate among treated hypertensive patients by region, $(\mathbf{G})$ control rate by sex, $(\mathbf{H})$ control rate among treated hypertensive patients by sex. $\# p<0.05,{ }^{*} p<0.001$.

awareness, treatment, and control rates have been a formidable public problem and may speed up the health expenditure increase in northeast China, especially in rural areas.

A previous national survey in 2002 showed that rural areas had a significantly lower prevalence of hypertension compared to urban areas ${ }^{8}$ however, the urban-rural gap gradually narrowed during the period of $1993-2011,{ }^{25}$ and the China Hypertension Survey conducted from 2012 to 2015 showed that the prevalence of hypertension among rural adults was comparable to that of urban residents. ${ }^{26}$ What is of note is that the latest large-scale survey (China PEASE Million Persons Project) undertaken from 2014 to
2017 showed that the hypertension prevalence was slightly higher in rural than in urban areas, ${ }^{2}$ roughly concordant with our data. The hypertension prevalence rates in northeast China are similar to the prevalence reported from lowand lower-middle-income countries. The variances of urban-rural differences are likely related to more rapid increase of hypertension in the rural population, although the prevalence of hypertension increased both in urban and rural areas. 5,15

The Seventh Joint National Committee (JNC7) guidelines (2003) categorized individuals with SBP $\geq 120 \mathrm{mmHg}$ and $<140 \mathrm{mmHg}$ and DBP $\geq 80 \mathrm{mmHg}$ and $<90 \mathrm{mmHg}$ and not on antihypertensive medication as prehypertensive. ${ }^{27}$ 


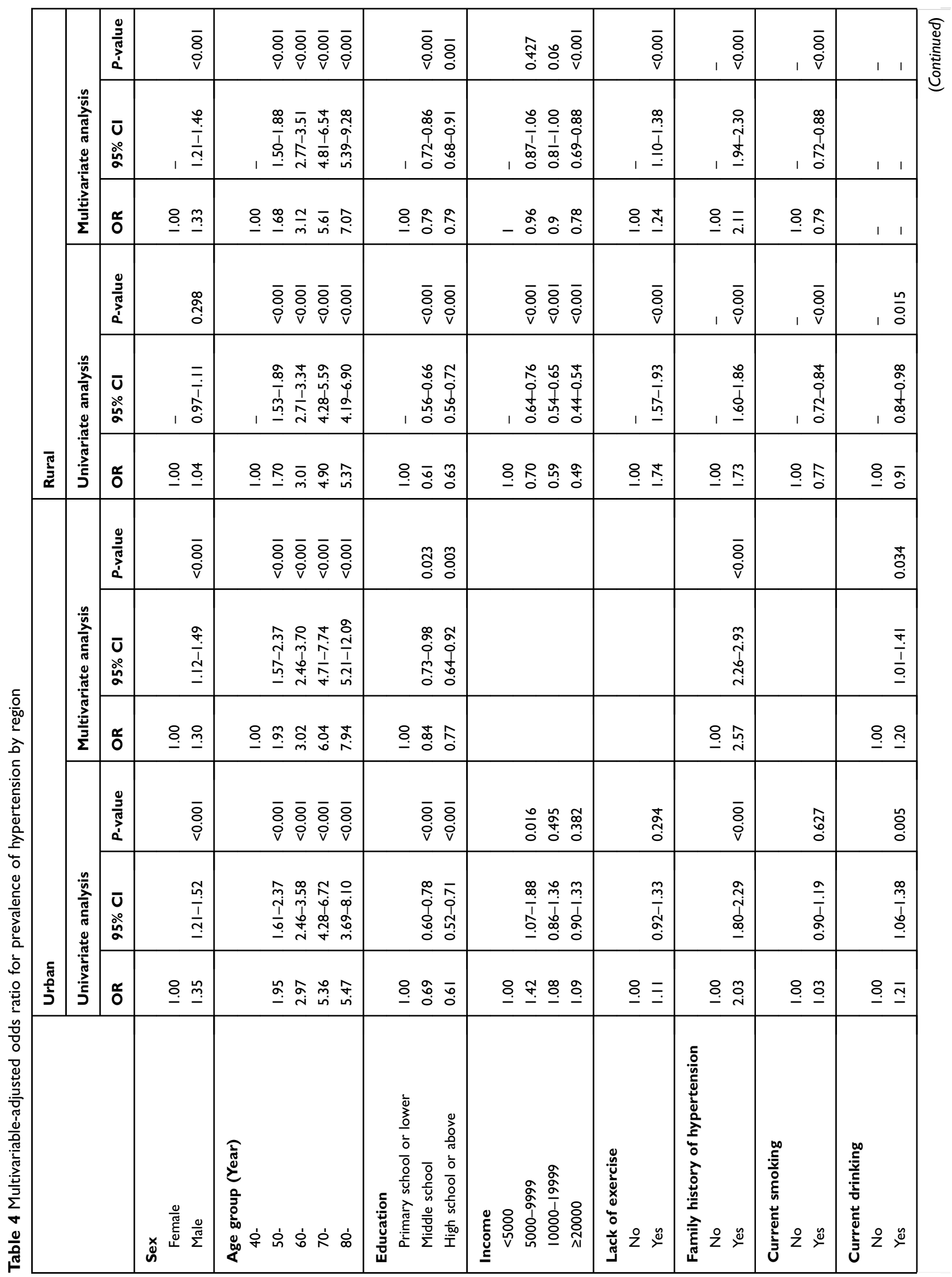




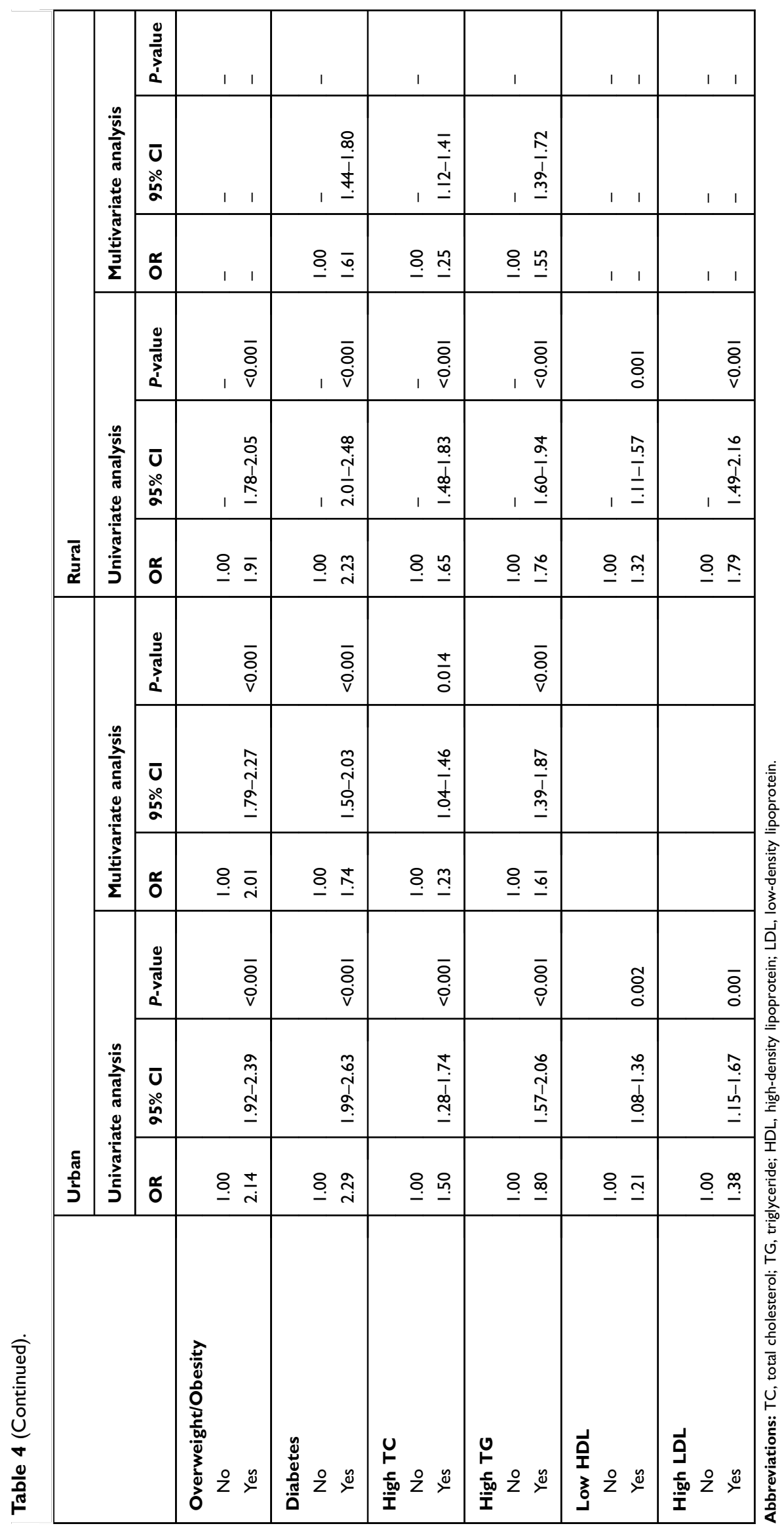




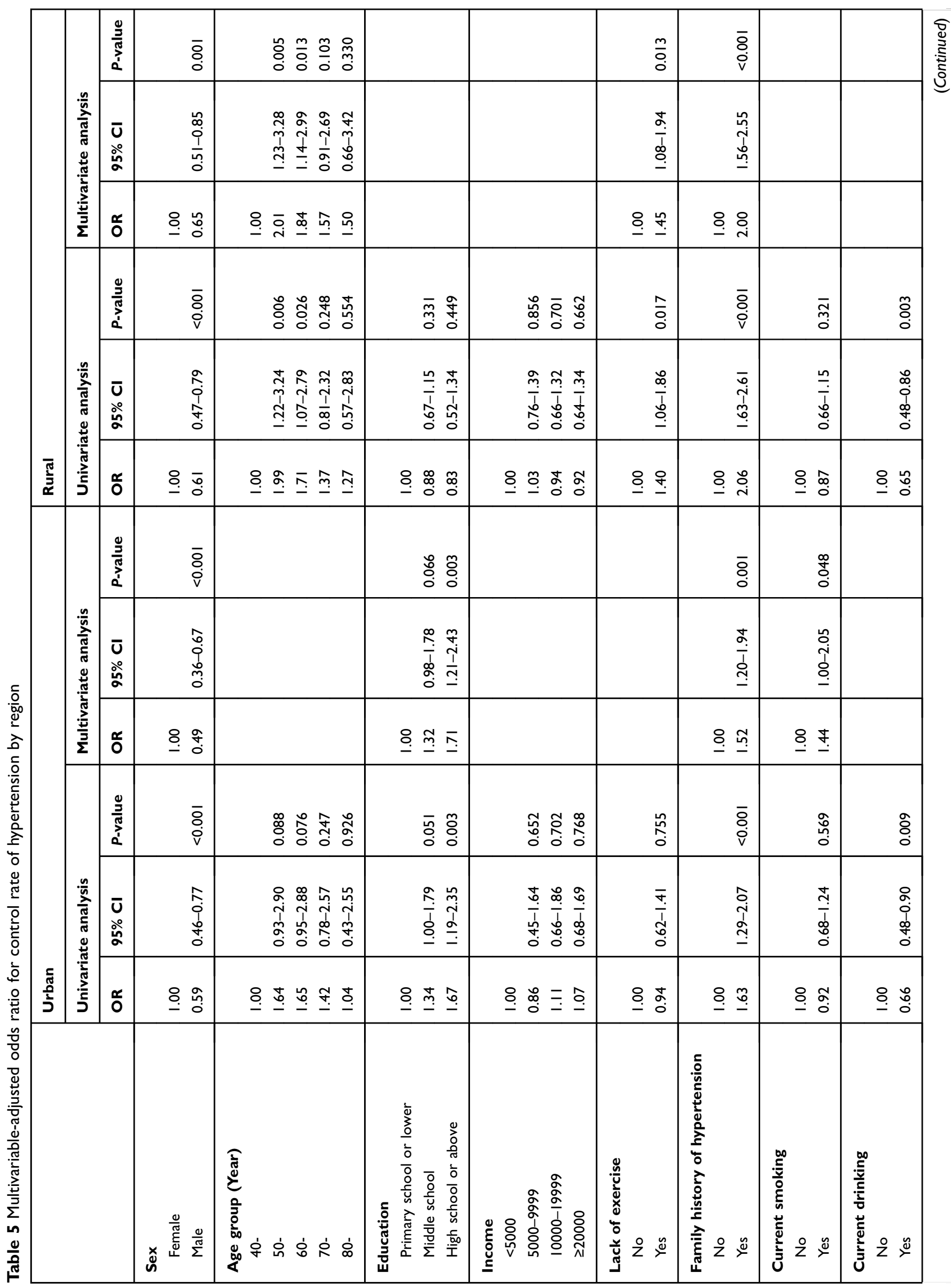




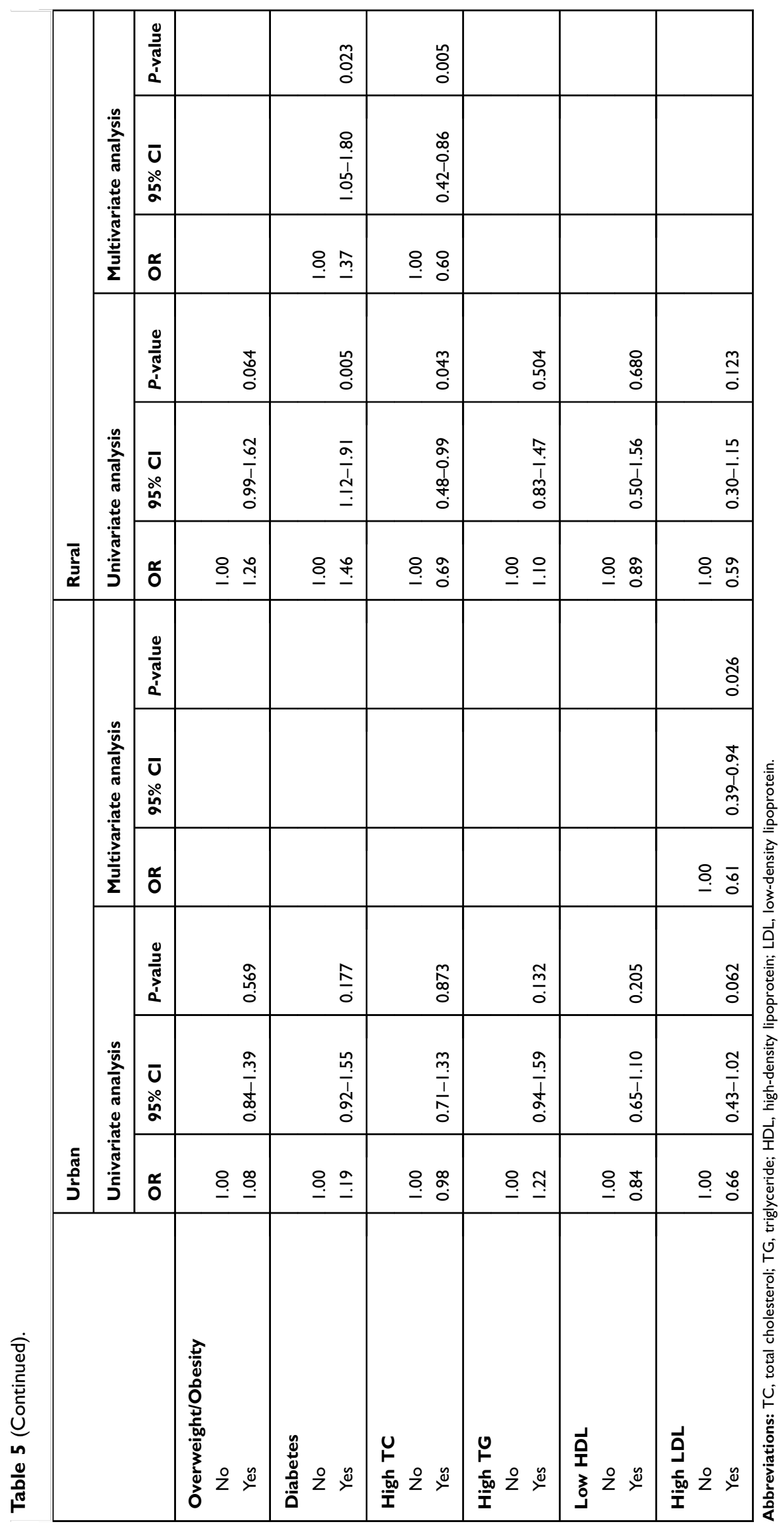



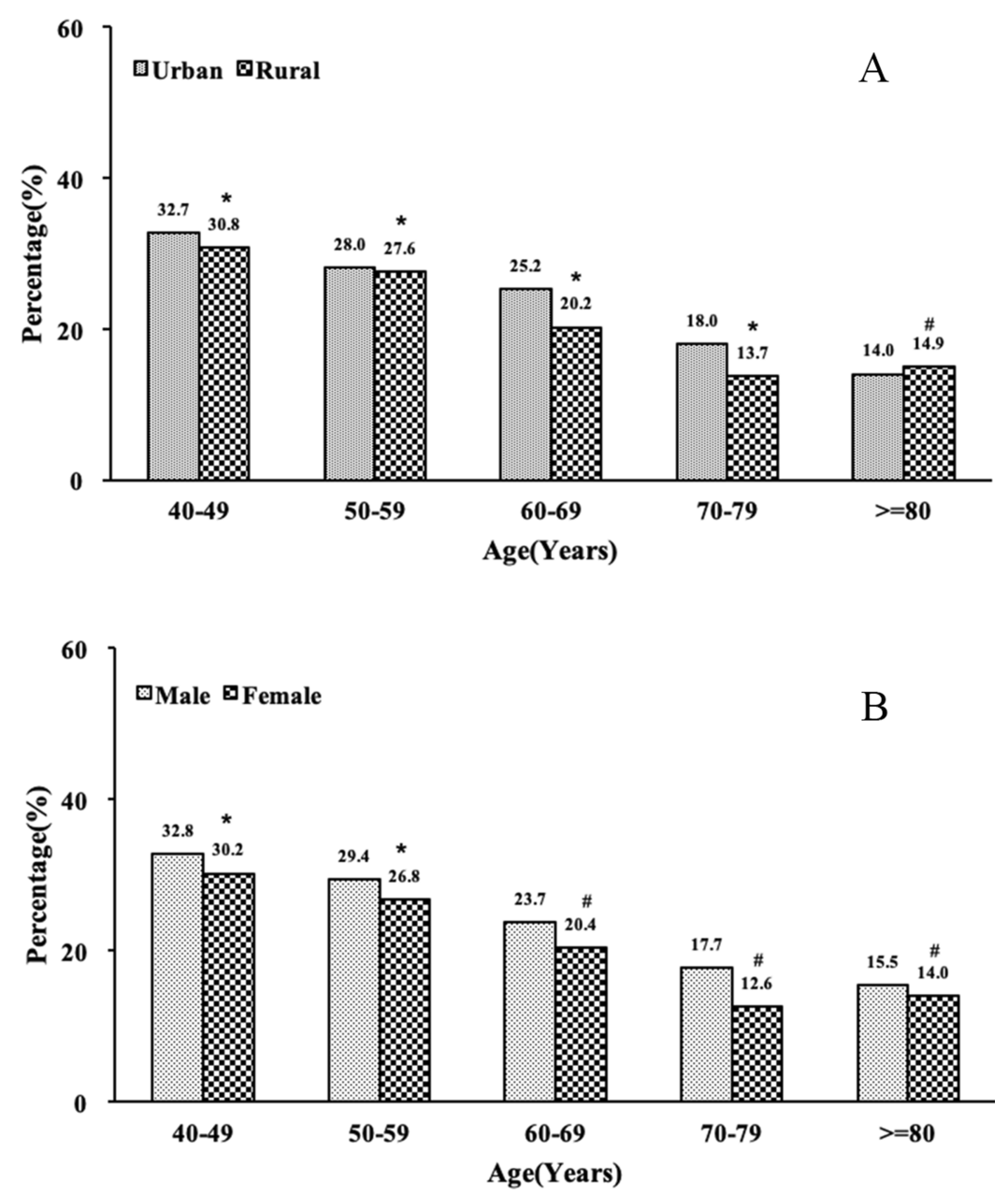

Figure 5 The absolute differences in prevalence of hypertension according to 2010 Chinese guidelines for the management of hypertension to $2017 \mathrm{ACC} / \mathrm{AHA}$ high blood pressure guidelines by region (A) and $\operatorname{sex}(\mathbf{B}){ }^{\#} \mathrm{p}<0.05,{ }^{*} \mathrm{p}<0.001$.

Individuals with prehypertension are associated with elevated overall cardiovascular risk compared to those with normal blood pressure $(<120 / 80 \mathrm{mmHg}) \cdot{ }^{28-30}$ Therefore, the new 2017 ACC/AHA guidelines on the prevention and treatment of hypertension have updated the definition of hypertension as well as the blood pressure treatment goal of 130/80 mmHg. ${ }^{18}$ Under the new guidelines, the prevalence of hypertension in northeast China increased nearly twothirds compared with the current Chinese guidelines $(80.6 \%$ vs $50.2 \%)$, which are significantly higher than the overall increased rate at the national level of China and the United States. ${ }^{26,31}$ Compared to the rural areas, the increase rate was higher in urban areas $(27.9 \%$ vs $25.7 \%, P<0.05)$. And, since the current Chinese guidelines still stipulate a treatment goal of 140/90 $\mathrm{mmHg}$, many of the categories previously known as prehypertension will now be defined as hypertension. Therefore, more attention should be paid to populations with prehypertension to avoid future worsening of premature cardiovascular disease.
The awareness, treatment, and control rates of hypertension in northeast China are far from satisfactory and remain significantly below those of developed countries as well as the national level. ${ }^{10,32,33}$ The status of hypertension in northeast China is worrisome. As hypertension risk factors involve sociological, environmental, and physical, to clarify the related modifiable risk factors of hypertension according to background characteristics including region and sex differences are essential for rational planning of health services.

A previous study advocated that urbanization was the major social determinants of hypertension in rural areas, ${ }^{34,35}$ however, our results revealed that in addition to the differences in prevalence and control rate of hypertension, the risk factors of hypertension in urban and rural areas varied widely. After adjusting the common traditional risk factors including advancing age, male gender, family history of hypertension, and low education in both urban and rural areas, lack of exercise has been the major risk factor for the 
Table 6 Prevalence and control rate according to 2010 Chinese high blood pressure guidelines and 2017 ACC/AHA high blood pressure guidelines

\begin{tabular}{|c|c|c|c|c|c|c|}
\hline \multirow[t]{2}{*}{ Characteristics } & \multicolumn{2}{|c|}{ Prevalence } & \multicolumn{2}{|l|}{ Control } & \multicolumn{2}{|c|}{$\begin{array}{l}\text { Control among treated } \\
\text { patients }\end{array}$} \\
\hline & $\begin{array}{l}2010 \\
\text { Chinese }\end{array}$ & $\begin{array}{l}2017 A C C I \\
\text { AHA }\end{array}$ & $\begin{array}{l}2010 \\
\text { Chinese }\end{array}$ & $\begin{array}{l}2017 A C C I \\
\text { AHA }\end{array}$ & $\begin{array}{l}2010 \\
\text { Chinese }\end{array}$ & $\begin{array}{l}2017 \text { ACCI } \\
\text { AHA }\end{array}$ \\
\hline Overall & 56.8 & 80.6 & 5.7 & 0.9 & 14.9 & 3.3 \\
\hline \multicolumn{7}{|l|}{ Age group } \\
\hline $40-49$ & 36.9 & 68.0 & 3.3 & 0.4 & 13.3 & 2.7 \\
\hline $50-59$ & 50.0 & 77.7 & 6.5 & 0.8 & 18.0 & 3.3 \\
\hline $60-69$ & 62.5 & 84.3 & 6.2 & I.I & 15.0 & 3.4 \\
\hline $70-79$ & 73.9 & 88.8 & 5.1 & 1.1 & 12.0 & 3.1 \\
\hline$\geq 80$ & 75.4 & 90.1 & 4.1 & 1.4 & 13.1 & 5.4 \\
\hline$P$-value & $<0.001$ & $<0.001$ & $<0.001$ & 0.021 & 0.005 & 0.668 \\
\hline \multicolumn{7}{|l|}{ Sex } \\
\hline Male & 58.8 & 78.4 & 4.1 & 0.5 & 13.6 & 2.6 \\
\hline Female & 55.6 & 84.0 & 6.8 & 1.1 & 15.6 & 3.7 \\
\hline$P$-value & $<0.001$ & $<0.001$ & $<0.001$ & $<0.001$ & 0.102 & 0.064 \\
\hline \multicolumn{7}{|l|}{ Education, n (\%) } \\
\hline Primary school or lower & 63.2 & 83.4 & 4.5 & 0.8 & 12.0 & 2.8 \\
\hline Middle school & 50.9 & 78.1 & 6.6 & 1.0 & 17.4 & 4.0 \\
\hline High school or above & 49.5 & 77.3 & 8.5 & 1.0 & 21.5 & 4.0 \\
\hline$P$-value & $<0.001$ & $<0.001$ & $<0.001$ & 0.382 & $<0.001$ & 0.081 \\
\hline \multicolumn{7}{|l|}{ Income, n (\%) } \\
\hline$<5000$ & 64.9 & 84.8 & 4.1 & 0.7 & 10.6 & 2.3 \\
\hline $5000-9999$ & 57.6 & 80.4 & 4.4 & 0.4 & 12.5 & 1.5 \\
\hline $10000-19999$ & 52.7 & 78.6 & 5.7 & 0.9 & 16.0 & 3.6 \\
\hline$\geq 20000$ & 50.0 & 77.3 & 8.7 & 1.4 & 21.5 & 5.5 \\
\hline$P$-value & $<0.001$ & $<0.001$ & $<0.001$ & $<0.001$ & $<0.001$ & $<0.001$ \\
\hline \multicolumn{7}{|l|}{ Mean BMI,kg/m² } \\
\hline$<18.5$ & 46.6 & 70.7 & 4.7 & 1.0 & 19.7 & 6.6 \\
\hline $18.5-23.9$ & 47.9 & 73.1 & 4.9 & 0.8 & 16.0 & 3.8 \\
\hline $24.0-27.9$ & 60.1 & 84.3 & 6.4 & I.I & 16.1 & 3.8 \\
\hline$\geq 28.0$ & 72.9 & 92.2 & 5.6 & 0.7 & 11.7 & 1.9 \\
\hline$P$-value & $<0.001$ & $<0.001$ & 0.034 & 0.209 & 0.005 & 0.011 \\
\hline \multicolumn{7}{|l|}{ Current smoking, \% } \\
\hline Yes & 53.9 & 79.9 & 4.9 & 0.8 & 16.6 & 4.2 \\
\hline No & 57.7 & 80.8 & 5.9 & 0.9 & 14.6 & 3.1 \\
\hline$P$-value & $<0.001$ & 0.155 & 0.049 & 0.687 & 0.158 & 0.135 \\
\hline \multicolumn{7}{|l|}{ Current drinking, $\%$} \\
\hline Yes & 56.9 & 82.8 & 3.9 & 0.5 & 14.7 & 2.7 \\
\hline No & 56.8 & 79.8 & 6.3 & 1.0 & 15.0 & 3.5 \\
\hline$P$-value & 0.842 & $<0.001$ & $<0.001$ & 0.001 & 0.842 & 0.269 \\
\hline \multicolumn{7}{|l|}{ Lack of exercise, \% } \\
\hline Yes & 66.8 & 86.1 & 5.7 & 1.0 & 12.4 & 2.7 \\
\hline No & 55.3 & 79.8 & 5.7 & 0.9 & 15.5 & 3.5 \\
\hline$P$-value & $<0.001$ & & 0.974 & 0.653 & 0.031 & 0.321 \\
\hline
\end{tabular}

(Continued) 
Table 6 (Continued).

\begin{tabular}{|c|c|c|c|c|c|c|}
\hline \multirow[t]{2}{*}{ Characteristics } & \multicolumn{2}{|c|}{ Prevalence } & \multicolumn{2}{|l|}{ Control } & \multicolumn{2}{|c|}{$\begin{array}{l}\text { Control among treated } \\
\text { patients }\end{array}$} \\
\hline & $\begin{array}{l}2010 \\
\text { Chinese }\end{array}$ & $\begin{array}{l}2017 A C C / \\
\text { AHA }\end{array}$ & $\begin{array}{l}2010 \\
\text { Chinese }\end{array}$ & $\begin{array}{l}2017 A C C / \\
\text { AHA }\end{array}$ & $\begin{array}{l}2010 \\
\text { Chinese }\end{array}$ & $\begin{array}{l}2017 A C C / \\
\text { AHA }\end{array}$ \\
\hline $\begin{array}{l}\text { Family history of hypertension, \% } \\
\text { Yes } \\
\text { No } \\
\text { P-value }\end{array}$ & $\begin{array}{l}66.5 \\
52.1 \\
<0.001\end{array}$ & $\begin{array}{l}86.4 \\
77.8 \\
<0.001\end{array}$ & $\begin{array}{l}7.6 \\
4.5 \\
<0.001\end{array}$ & $\begin{array}{l}1.2 \\
0.7 \\
0.001\end{array}$ & $\begin{array}{l}14.8 \\
|5 .| \\
0.78 \mid\end{array}$ & $\begin{array}{l}3.1 \\
3.6 \\
0.438\end{array}$ \\
\hline $\begin{array}{l}\text { Region } \\
\text { Urban } \\
\text { Rural } \\
\text { P-value }\end{array}$ & $\begin{array}{l}50.9 \\
59.2 \\
<0.001\end{array}$ & $\begin{array}{l}76.6 \\
82.2 \\
<0.001\end{array}$ & $\begin{array}{l}11.4 \\
3.7 \\
<0.001\end{array}$ & $\begin{array}{l}1.8 \\
0.6 \\
<0.001\end{array}$ & $\begin{array}{l}24.4 \\
10.5 \\
<0.001\end{array}$ & $\begin{array}{l}5.6 \\
2.2 \\
<0.001\end{array}$ \\
\hline $\begin{array}{l}\text { Diabetes } \\
\text { Yes } \\
\text { No } \\
P \text {-value }\end{array}$ & $\begin{array}{l}71.7 \\
53.7 \\
<0.001\end{array}$ & $\begin{array}{l}90.2 \\
78.6\end{array}$ & $\begin{array}{l}7.4 \\
5.2 \\
<0.001\end{array}$ & $\begin{array}{l}1.6 \\
0.7 \\
<0.001\end{array}$ & $\begin{array}{l}\mid 4.5 \\
\mid 5.2 \\
0.57 \mid\end{array}$ & $\begin{array}{l}3.8 \\
3.1 \\
0.261\end{array}$ \\
\hline $\begin{array}{l}\text { High TC } \\
\text { Yes } \\
\text { No } \\
\text { P-value }\end{array}$ & $\begin{array}{l}66.2 \\
55.2 \\
<0.001\end{array}$ & $\begin{array}{l}87.3 \\
79.5 \\
<0.001\end{array}$ & $\begin{array}{l}5.0 \\
5.8 \\
0.159\end{array}$ & $\begin{array}{l}0.9 \\
0.9 \\
0.778\end{array}$ & $\begin{array}{l}12.6 \\
15.5 \\
0.053\end{array}$ & $\begin{array}{l}3.2 \\
3.4 \\
0.764\end{array}$ \\
\hline $\begin{array}{l}\text { High TG } \\
\text { Yes } \\
\text { No } \\
\text { P-value }\end{array}$ & $\begin{array}{l}67.6 \\
54.5 \\
<0.001\end{array}$ & $\begin{array}{l}88.8 \\
78.8 \\
<0.001\end{array}$ & $\begin{array}{l}6.7 \\
5.4 \\
0.02\end{array}$ & $\begin{array}{l}1 \\
0.9 \\
0.419\end{array}$ & $\begin{array}{l}15.1 \\
14.9 \\
0.875\end{array}$ & $\begin{array}{l}3 \\
3.4 \\
0.532\end{array}$ \\
\hline $\begin{array}{l}\text { Low HDL } \\
\text { Yes } \\
\text { No } \\
\text { P-value }\end{array}$ & $\begin{array}{l}57.4 \\
56.7 \\
0.512\end{array}$ & $\begin{array}{l}82.1 \\
80.4 \\
0.06\end{array}$ & $\begin{array}{l}8.0 \\
5.4 \\
<0.001\end{array}$ & $\begin{array}{l}1.4 \\
0.8 \\
0.025\end{array}$ & $\begin{array}{l}17.5 \\
14.6 \\
0.074\end{array}$ & $\begin{array}{l}4.2 \\
3.2 \\
0.201\end{array}$ \\
\hline $\begin{array}{l}\text { High LDL } \\
\text { Yes } \\
\text { No } \\
\text { P-value }\end{array}$ & $\begin{array}{l}65.3 \\
56.3 \\
<0.001\end{array}$ & $\begin{array}{l}86 \\
80.3 \\
<0.001\end{array}$ & $\begin{array}{l}4.7 \\
5.8 \\
0.268\end{array}$ & $\begin{array}{l}0.7 \\
0.9 \\
0.431\end{array}$ & $\begin{array}{l}10.9 \\
15.3 \\
0.038\end{array}$ & $\begin{array}{l}2 \\
3.4 \\
0 .|7|\end{array}$ \\
\hline
\end{tabular}

Abbreviations: BMI, body mass index; TC, total cholesterol; TG, triglyceride; HDL, high-density lipoprotein; LDL, low-density lipoprotein.

prevalence of hypertension in rural areas. Reasons for this may be the cool climate and lack of exercise facilities in northeast rural China. On the other hand, overweight/obesity and current drinking were related to increased risk of hypertension in urban areas; therefore, lifestyle change should be highlighted in urban areas.

Despite the geographic variation, the differences between males and females were observed in the study. Males were more likely to have hypertension and poor control than females, which coincided with other regions in China and other countries, as previously reported. ${ }^{36}$ However, compared to men, lack of exercise was a contributor to the high prevalence of hypertension in females. Moreover, a man with a higher income was less likely to have hypertension, and men with relatively lower income were likely to experience occupational and relationship stress, together with an unhealthy lifestyle. In addition, males had a higher change than females according to the 2017 ACC/AHA guidelines, due to the large proportion of prehypertension in males. Therefore, region- and sex-specific strategies in the prevention of hypertension, particularly for men, could yield great improvement.

The strength of this study is that the survey was conducted in a representative population including rural and 
urban populations and was adequately powered, thus providing opportunity to comprehensively assess the current hypertension epidemic. The major limitation of this study was that the sample size in the urban area was relatively small, since most of the residents in Liaoning Province as well as China still live in rural areas. Moreover, the risks of prehypertension and hypertension including salt intake were not evaluated, as it was well established in recent years as a major contributor to hypertension. ${ }^{3,37}$ Furthermore, the potential impact of the 2017 ACC/AHA guidelines on the prevention and management of hypertension should be fully assessed. In addition, the high percentage of men in the floating population in China might responsible for the high proportion of women in the study. ${ }^{38}$ However, as women were more sensitive to health education and had better access to prevention strategies, selection bias was inevitable in the study. ${ }^{39}$ In this study, all comparisons were based on the standardized population, which minimizes selection bias.

In conclusion, the high prevalence, but low awareness, treatment, and control rates of hypertension present a considerable population burden both in urban and rural areas of northeast China. However, in rural areas, the burden is more significant. Moreover, the elevated mean blood pressure levels and high prevalence of prehypertension remain worrisome. Under the 2017 ACC/AHA guidelines, the prevalence of hypertension significantly increased, whereas the control rate markedly decreased; however, the change rate in urban areas was more predominate because of the high prevalence of prehypertension. Furthermore, the risk factors of hypertension in urban and rural areas differ widely; the lack of exercise was associated with high prevalence of hypertension in rural areas, whereas overweight/obesity and current drinking contributed to the development of hypertension in urban areas. Therefore, hypertension has been becoming a major public health challenge in northeast China; region-specific strategies between urban and rural areas should be highlighted in northeast China, in addition to adequate screening and education on the prevention and management of hypertension.

\section{Acknowledgment}

This study was supported by the Department of Science and Technology of Liaoning Province (2018225065) and the National Natural Science Foundation of China (81701699). We would like to thank the CDC of Chaoyang, Liaoyang, Dandong, and Donggang city in Liaoning Province, which worked hard to ensure the reliability and accuracy of data.

\section{Disclosure}

The authors report no conflicts of interest in this work.

\section{References}

1. Collaborators GBDRF. Global, regional, and national comparative risk assessment of 79 behavioural, environmental and occupational, and metabolic risks or clusters of risks, 1990-2015: a systematic analysis for the global burden of disease study 2015. Lancet. 2016;388:1659-1724. doi:10.1016/S0140-6736(16)31679-8.

2. Lu J, Lu Y, Wang X, et al. Prevalence, awareness, treatment, and control of hypertension in China: data from 1.7 million adults in a population-based screening study (China PEACE million persons project). Lancet. 2017;390:2549-2558. doi:10.1016/S0140-6736(17) 32478-9

3. Qi Y, Han X, Zhao D, et al. Long-term cardiovascular risk associated with stage 1 hypertension defined by the 2017 ACC/AHA hypertension guideline. J Am Coll Cardiol. 2018;72:1201-1210. doi:10.1016/ j.jacc.2018.06.056

4. Satoh A, Arima H, Ohkubo T, et al.; Group NDR. Associations of socioeconomic status with prevalence, awareness, treatment, and control of hypertension in a general Japanese population: NIPPON DATA2010. J Hypertens. 2017;35:401-408. doi:10.1097/HJH.0000000000001169

5. Li Z, Guo X, Zheng L, Yang H, Sun Y. Grim status of hypertension in rural China: results from Northeast China rural cardiovascular health study 2013. J Am Soc Hypertens. 2015;9:358-364. doi:10.1016/j. jash.2015.02.014

6. Yan $\mathrm{R}$, Li W, Yin $\mathrm{L}$, Wang $\mathrm{Y}$, Bo J; Investigators PU-C. Cardiovascular diseases and risk-factor burden in urban and rural communities in high-, middle-, and low-income regions of China: a large community-based epidemiological study. J Am Heart Assoc. 2017;6:e004445. doi:10.1161/JAHA.116.004445

7. Wang W, Jiang B, Sun H, et al.; Investigators NE-C. Prevalence, incidence, and mortality of stroke in China: results from a nationwide population-based survey of 480,687 adults. Circulation. 2017;135:759771. doi:10.1161/CIRCULATIONAHA.116.025250

8. Wu Y, Huxley R, Li L, et al. China NSC and China NWG. Prevalence, awareness, treatment, and control of hypertension in China: data from the China National Nutrition and Health Survey 2002. Circulation. 2008;118:2679-2686. doi:10.1161/CIRCULATIONAHA.108.788166

9. Zhang FL, Guo ZN, Xing YQ, Wu YH, Liu HY, Yang Y. Hypertension prevalence, awareness, treatment, and control in northeast China: a population-based cross-sectional survey. J Hum Hypertens. 2017;32:54-65. doi:10.1038/s41371-017-0003-4

10. Zhang Y, Moran AE. Trends in the prevalence, awareness, treatment, and control of hypertension among young adults in the United States, 1999 to 2014. Hypertension. 2017;70:736-742. doi:10.1161/ HYPERTENSIONAHA.117.09801

11. Booth JN 3rd, Li J, Zhang L, Chen L, et al. Trends in prehypertension and hypertension risk factors in US adults: 1999-2012. Hypertension. 2017;70:275-284. doi:10.1161/HYPERTENSIONAHA.116.09004

12. Joshi SR, Shah SN. Control of blood pressure in India: rule of halves still very much valid. J Assoc Physicians India. 2003;51:151-152.

13. Feng XL, Pang M, Beard J. Health system strengthening and hypertension awareness, treatment and control: data from the China health and retirement longitudinal study. Bull World Health Organ. 2014;92:29-41. doi:10.2471/BLT.13.124495

14. Lu Y, Wang P, Zhou T, et al. Comparison of prevalence, awareness, treatment, and control of cardiovascular risk factors in China and the United States. J Am Heart Assoc. 2018;7:e007462. doi:10.1161/ JAHA. 118.008528

15. Xing L, Liu S, Tian Y, et al. Trends in status of hypertension in rural northeast China: results from two representative cross-sectional surveys, 2013-2018. J Hypertens. 2019;28:1400-1408. 
16. Xing L, Jing L, Tian Y, et al. High prevalence of stroke and uncontrolled associated risk factors are major public health challenges in rural northeast China: a population-based study. Int J Stroke. 2019; May 15. doi:10.1177/1747493019851280

17. Liu LS, Gao RL, Chen WW, et al. 2010 Chinese guidelines for the management of hypertension. Zhonghua Xin Xue Guan Bing Za Zhi. 2011;39:579-615.

18. Whelton PK, Carey RM, Aronow WS, et al. 2017 ACC/AHA/AAPA/ $\mathrm{ABC} / \mathrm{ACPM} / \mathrm{AGS} / \mathrm{APhA} / \mathrm{ASH} / \mathrm{ASPC}$ NMA/PCNA Guideline for the prevention, detection, evaluation, and management of high blood pressure in adults: executive summary: a report of the American College of Cardiology/American Heart Association Task Force on Clinical Practice Guidelines. Hypertension. 2018;71:1269-1324. doi:10.1161/HYP.000 0000000000066

19. Yan L, Xu MT, Yuan L, et al. Prevalence of dyslipidemia and its control in type 2 diabetes: a multicenter study in endocrinology clinics of China. J Clin Lipidol. 2016;10:150-160. doi:10.1016/j.jacl.2015.10.009

20. Chamberlain JJ, Rhinehart AS, Shaefer CF Jr., Neuman A. Diagnosis and management of diabetes: synopsis of the 2016 American Diabetes Association standards of medical care in diabetes. Ann Intern Med. 2016;164:542-552. doi:10.7326/M15-3016

21. Wang J, Ning X, Yang L, et al. Trends of hypertension prevalence, awareness, treatment and control in rural areas of northern China during 1991-2011. J Hum Hypertens. 2014;28:25-31. doi:10.1038/jhh.2013.44

22. Nishida C, Ko GT, Kumanyika S. Body fat distribution and noncommunicable diseases in populations: overview of the $2008 \mathrm{WHO}$ expert consultation on waist circumference and waist-hip ratio. Eur J Clin Nutr. 2010;64:2-5. doi:10.1038/ejcn.2009.139

23. Collaborators GBDM. Global, regional, and national under-5 mortality, adult mortality, age-specific mortality, and life expectancy, 1970-2016: a systematic analysis for the global burden of disease study 2016. Lancet. 2017;390:1084-1150. doi:10.1016/S0140-6736(17)31833-0

24. Huang X, Zhou Z, Liu J, et al. Prevalence, awareness, treatment, and control of hypertension among China's Sichuan Tibetan population: a cross-sectional study. Clin Exp Hypertens. 2016;38:457-463. doi:10.3109/10641963.2016.1163369

25. Li J, Shi L, Li S, Xu L, Qin W, Wang H. Urban-rural disparities in hypertension prevalence, detection, and medication use among Chinese adults from 1993 to 2011. Int J Equity Health. 2017;16:50. doi:10.1186/s12939-017-0545-7

26. Wang Z, Chen Z, Zhang L, et al.; China Hypertension Survey I. Status of hypertension in China: results from the China Hypertension Survey, 2012-2015. Circulation. 2018;137:2344-2356. doi:10.1161/ CIRCULATIONAHA.117.032380

27. Chobanian AV, Bakris GL, Black HR, et al.; National Heart L, Blood Institute Joint National Committee on Prevention DE, Treatment of High Blood P and National High Blood Pressure Education Program Coordinating C. The seventh report of the Joint National Committee on Prevention, Detection, Evaluation, and Treatment of High Blood Pressure: the JNC 7 report. JAMA. 2003;289:2560-2572. doi:10.1001/jama.289.19.2560
28. Rahman M, Zaman MM, Islam JY, et al. Prevalence, treatment patterns, and risk factors of hypertension and pre-hypertension among Bangladeshi adults. J Hum Hypertens. 2018;32:334-348. doi:10.1038/s41371-017-0018-x

29. Piaditis G, Markou A, Papanastasiou LA II, Kaltsas G. Progress in aldosteronism: a review of the prevalence of primary aldosteronism in pre-hypertension and hypertension. Eur J Endocrinol. 2015;172: R191-R203. doi:10.1530/EJE-14-0537

30. Kanegae H, Oikawa T, Kario K. Should pre-hypertension be treated? Curr Hypertens Rep. 2017;19:91. doi:10.1007/s11906-017-0789-z

31. Khera R, Lu Y, Lu J, et al. Impact of 2017 ACC/AHA guidelines on prevalence of hypertension and eligibility for antihypertensive treatment in United States and China: nationally representative cross sectional study. BMJ. 2018;362:k2357. doi:10.1136/bmj. k2357

32. Scheltens T, Bots ML, Numans ME, et al. Awareness, treatment and control of hypertension: the 'rule of halves' in an era of risk-based treatment of hypertension. J Hum Hypertens. 2007;21:99-106. doi:10.1038/sj.jhh.1002123

33. Joffres M, Falaschetti E, Gillespie C, et al. Hypertension prevalence, awareness, treatment and control in national surveys from England, the USA and Canada, and correlation with stroke and ischaemic heart disease mortality: a cross-sectional study. BMJ Open. 2013;3: e003423. doi:10.1136/bmjopen-2013-003423

34. Reynolds K, Gu D, Muntner P, et al. Geographic variations in the prevalence, awareness, treatment and control of hypertension in China. J Hypertens. 2003;21:1273-1281. doi:10.1097/01.hjh.0000 059043.65882 .15

35. Chow CK, Teo KK, Rangarajan S, et al.; investigators PS. Prevalence, awareness, treatment, and control of hypertension in rural and urban communities in high-, middle-, and low-income countries. JAMA. 2013;310:959-968. doi:10.1001/jama.2013. 184182

36. Agyemang C, Nyaaba G, Beune E, et al. Variations in hypertension awareness, treatment, and control among Ghanaian migrants living in Amsterdam, Berlin, London, and nonmigrant Ghanaians living in rural and urban Ghana - the RODAM study. $J$ Hypertens. 2018;36:169-177. doi:10.1097/HJH.0000000000001520

37. Wu J, Li T, Song X, et al. Prevalence and distribution of hypertension and related risk factors in Jilin Province, China 2015: a cross-sectional study. BMJ Open. 2018;8:e020126. doi:10.1136/bmjopen2017-020126

38. statistics PsRoCNBo. People's Republic of China 2015 National Economic and Social Development Statistical Bulletin People's Daily. 2016-03-01:010.

39. Barker-Collo S, Bennett DA, Krishnamurthi RV, et al.; Group GBDW and Group GBDSPE. Sex differences in stroke incidence, prevalence, mortality and disability-adjusted life years: results from the global burden of disease study 2013. Neuroepidemiology. 2015;45:203-214. doi:10.1159/000441103
Clinical Epidemiology

\section{Publish your work in this journal}

Clinical Epidemiology is an international, peer-reviewed, open access, online journal focusing on disease and drug epidemiology, identification of risk factors and screening procedures to develop optimal preventative initiatives and programs. Specific topics include: diagnosis, prognosis, treatment, screening, prevention, risk factor modification,

Submit your manuscript here: https://www.dovepress.com/clinical-epidemiology-journal systematic reviews, risk \& safety of medical interventions, epidemiology \& biostatistical methods, and evaluation of guidelines, translational medicine, health policies \& economic evaluations. The manuscript management system is completely online and includes a very quick and fair peer-review system, which is all easy to use. 